\title{
NONLOCAL INTERACTION EQUATIONS IN ENVIRONMENTS WITH HETEROGENEITIES AND BOUNDARIES
}

\author{
LIJIANG WU AND DEJAN SLEPČEV
}

\begin{abstract}
We study well-posedness of a class of nonlocal interaction equations with spatially dependent mobility. We also allow for the presence of boundaries and external potentials. Such systems lead to the study of nonlocal interaction equations on subsets $\mathcal{M}$ of $\mathbb{R}^{d}$ endowed with a Riemannian metric $g$. We obtain conditions, relating the interaction potential and the geometry, which imply existence, uniqueness and stability of solutions. We study the equations in the setting of gradient flows in the space of probability measures on $\mathcal{M}$ endowed with Riemannian 2-Wasserstein metric.
\end{abstract}

\section{INTRODUCTION}

Nonlocal interaction equations serve as basic models of biological aggregation, that is collective motion of agents under influence of long-range interactions (via sight, sound, etc.). Their basic properties [42, 43, 32, 15, 11, 13, blowup (concentration) [10, 8, 7, 28, 30, 9, confinement [14, 5], stability and properties of stationary states [21, 22, 18, 23, 31, 4, asymptotic behavior [12, 33, 39, 29] and related models that incorporate further effects 6, 44 have been extensively studied. In this paper we investigate the nonlocal interaction equation in heterogeneous environments and also allow for the presence of domain boundaries. On the whole space (when no boundaries are present) the equations are of the form

$$
\frac{\partial}{\partial t} \mu(t, x)-\operatorname{div}(\mu(t, x) A(x) \nabla(W * \mu(t)(x)+V(x)))=0,
$$

where $\mu$ describes the agent density, $A$ is the mobility matrix (symmetric and positive definite), $W$ is the interaction potential and $V$ is the external potential.

The mobility endows the subsets of $\mathbb{R}^{d}$ with Riemannian structure, which leads us to study nonlocal interaction equations on manifolds. We study the well-posedness of the equations in the setting of gradient flows in spaces of probability measures [2, 13. To extend this setting to manifolds with boundary we needed to overcome several challenges. Namely "mass" can accumulate at the boundary and the velocities associated to the gradient flow are not continuous at the boundary. This also causes the problem that in general, we do not have the existence of optimal maps and thus we have to work with optimal plans instead. Furthermore the velocities (of the gradient flows) lack the stability properties used to prove the lower semicontinuity of the slope (see for example Lemma 2.7 in [13). Studying the equation on a manifold raises issues too. The curvature of the space can cause even the quadratic potential not to be geodesically semi-convex. Thus a particular care (and extra conditions) are needed when discussing properties like geodesic semi-convexity of energies. Furthermore many standard tools used to study nonlocal equations rely on the linearity of the underlying space and ability to directly identify tangent spaces at different points. Thus these tools do not readily transfer to the manifold setting. For example the standard proof of the characterization of the subdifferential of the interaction energy does not apply in the manifold setting. We develop alternative proofs to handle these challenges.

Date: October 31, 2013. 
1.0.1. Motivation. The studies of the nonlocal equations on heterogeneous environments are in part motivated by the desire to understand mechanisms which give rise to rolling swarms. Such swarms are observed in a number biological swarms, notably the locust swarms (see [41] and references therein). In [41, Topaz, Bernoff, Logan and Toolson propose a model which has a gradient flow structure of an energy that combines the interaction energy and potential energy terms (to model gravity and wind). The mobility in their system is as follows: consider the upper half plan $\mathbb{R}_{+}^{2}=$ $\left\{x=\left(x_{1}, x_{2}\right) \in \mathbb{R}^{2}: x_{2} \geq 0\right\}$. Above the ground the mobility is constant $\left(A(x)=I_{2 \times 2}\right)$, while on the ground the mobility in the horizontal direction if zero $(A(x)=\operatorname{diag}(0,1))$. They conduct numerical experiments and observe rolling swarms. Here we introduce a model where the change in mobility is more gradual, and thus amenable to rigorous study. The solutions still exhibit the rolling swarms when a smoothed out version of the mobility in [41] is considered. Moreover, rolling forms are present if the horizontal mobility is stratified (increases with height), even if gravity is not present. Figure 1 illustrates such a rolling swarm. The interaction potential used is among ones considered in [31, and is given by $W(z)=w(|z|)$ with $w^{\prime}(r)=\tanh (3(1-r))+0.3$. On the right, we also show the corresponding traveling "swarm" in the homogeneous environment. The velocities of all particles are the same. Moreover the configuration seen in the moving coordinate frame is a steady state of the energy $\mathcal{E}_{2}(\mu)=\iint W(x-y) d \mu(x) d \mu(y)$.
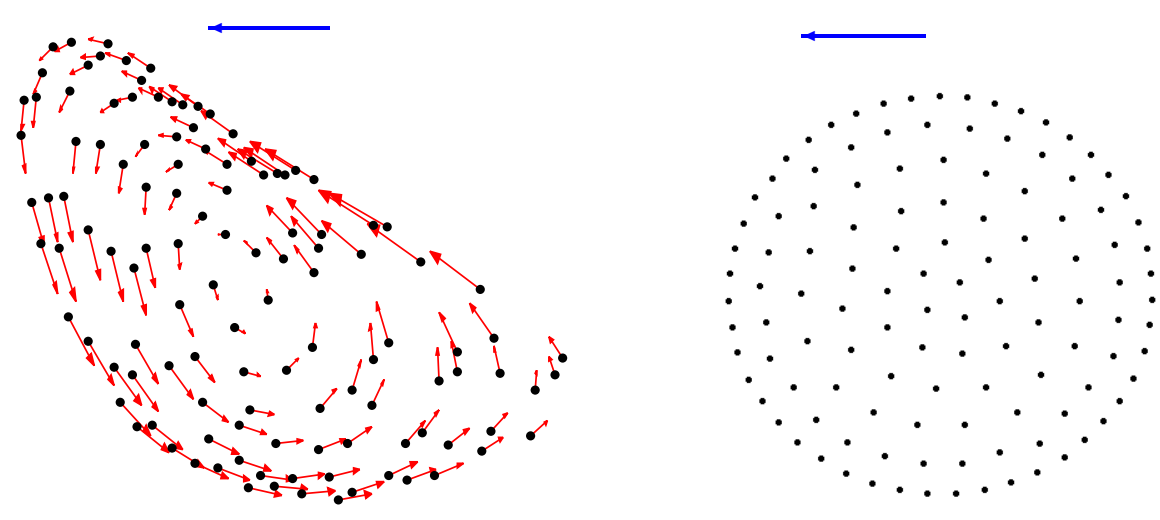

Figure 1. Consider gradient flows of $\mathcal{E}(\mu)=\iint W(x-y) d \mu(x) d \mu(y)+\int x_{1} d \mu(x)$, with respect to a stratified metric $G(x)=G\left(\left(x_{1}, x_{2}\right)\right)=\operatorname{diag}\left(\frac{1}{x_{2}^{2}}, 1\right)$ and Euclidean metric. The gradient flow with respect to the stratified metric admits a rolling-wave solution made of a finite number of particles (left). The solution is given in the reference frame of the center of mass. The overall direction of motion is indicated by the large arrow on top (blue). The smaller arrows indicate the velocity of particles in the moving coordinates. The gradient flow with respect to Euclidean metric admits a traveling wave (right). All particle velocities are the same; hence in the moving coordinates the solution appears stationary.

1.0.2. Gradient flows in spaces of probability measures on manifolds: Background. Let us first recall that the existence of optimal transportation maps on manifolds was first considered by McCann [35, and subsequently generalized in [16, 20]. The regularity of these maps has been the subject of a lot of recent activity and progress (see [24] and references therein). For our purposes however, the results on optimal transportation plans presented in Villani's book [45] are sufficient. 
Regarding the gradient flows there has been a significant progress in investigating the gradient flow of entropy (i.e. the heat equation) and other internal energies on manifolds, as well spaces with weaker geometric structure. In particular Lisini 34 considered $\mathbb{R}^{d}$ endowed with a bounded Riemannian metric $G$, satisfying $\Lambda_{1} I_{d} \leq G \leq \Lambda_{2} I_{d}$, and showed the existence of solutions to the equation

$$
\frac{\partial}{\partial t} u(t, x)-\operatorname{div}(A(x)[\nabla(f(u(t, x))+u(t, x) \nabla V(x)])=0,
$$

on the whole space $\mathbb{R}^{d}$ with $A(x)=G^{-1}(x)$. In 37] Otto and Westdickenberg used an Eulerian calculus method to give sufficient conditions for the internal and potential energy to be geodesically convex in the space of probability measures endowed with Riemannian Wasserstein metric. In [17, Daneri and Saveré refined the approach of [37] to include the case of geodesic semi-convexity. In [40, Sturm gave the necessary and sufficient conditions for internal and potential energies to be $\lambda$-geodesically convex in the space of probability measures endowed with Riemannian Wasserstein metric. Erbar [19] used these conditions to establish well-posedness of heat equation on manifolds in the framework of gradient flows in spaces of probability measures. Gradient flows of the internal energy on manifolds were also discussed in in 45. Connections with geometry and extensions to weaker spaces have received significant attention, see [3, 26, 27, 36] and references therein. However, to the best of our knowledge the gradient flow of nonlocal interaction energies on manifolds has not been considered.

1.1. Description of the problem. Let $\mathcal{M}$ be a, possibly unbounded, $d$-dimensional subset of $\mathbb{R}^{d}$ with $C^{2}$ boundary. We consider $\mathcal{M}$ with a Riemannian metric $g$. Throughout the paper we assume that $(\mathcal{M}, g)$ is complete under the metric induced by $g$ and geodesically convex, that is, for any two points in $\mathcal{M}$ there exists a length minimizing geodesic in $\mathcal{M}$ connecting them. The Riemannian structure encodes the mobility of the agents which depends on the environment. The strength of the interaction is not affected by the environment. To give an example, we study situations where the properties of the terrain affect the mobility of the agents, but not their ability to see each other. Also the density of agents at a given location is with respect to the standard Euclidean volume/area; it is not affected by the metric $g$. This leads us to study equations in a mixed formulation, where the volume and interaction are with respect to Euclidean structure, while the mobility is with respect to the manifold structure, $g$.

To study the equations we use their gradient-flow structure, which enables us to write the equations in the form that at the same time applies both to discrete systems with finitely many agents and continuum descriptions. This follows from the theory developed for studies of the nonlocal interaction equations in homogeneous environments 2, 13. More precisely a configuration (distribution of agents) is described by a measure $\mu$ supported on $\mathcal{M}$. The system is assumed to be conservative in the sense that no agents are created or leave the system during the evolution. In other words $\mu(\mathcal{M})$ does not change in time. This allows us to, by renormalizing the problem if needed, assume that configurations $\mu$ are probability measures.

The interaction is described by a symmetric interaction potential $W$. The corresponding interaction energy is

$$
\mathcal{W}(\mu)=\frac{1}{2} \int_{\mathcal{M} \times \mathcal{M}} W(x-y) d \mu(x) d \mu(y) .
$$

In addition to interaction we model the environmental influences such as gravity or food distribution by a potential $V$, which defines the potential energy

$$
\mathcal{V}(\mu)=\int_{\mathcal{M}} V(x) d \mu(x)
$$


The total energy is

$$
\mathcal{E}(\mu)=\mathcal{W}(\mu)+\mathcal{V}(\mu)
$$

1.1.1. Gradient flow structure. We introduce the geometry on the space of configurations first on a formal level. In nonlocal interaction equations (with no regularizing terms) mass can accumulate at the boundary and furthermore the velocity that describes the gradient flow can be discontinuous at the boundary. For this reason we use a more general way to introduce the gradient flow than is typically the case in heuristic arguments. We use a Lagrangian description of tangent vectors at a configuration. That is tangent vectors to the space of configurations are vector fields on $\mathcal{M}$. As is standard in differential geometry of manifolds with boundary, even at $x \in \partial \mathcal{M}$ we define the tangent space $T_{x} \mathcal{M}$ to be a vector space, in other words we do allow vectors that point outside the manifold. However since a path in the configuration space cannot take mass outside of $\mathcal{M}$, not all of the vectors in $T_{x} \mathcal{M}$ are admissible as values of the tangent vector field to the path in the configuration space. To define the set of admissible vectors for $x \in \partial \mathcal{M}$, let $T_{x}^{\text {in }} \mathcal{M}$ be the inward sector, namely the closed half-space of tangent vectors that do not point outside $\mathcal{M}$. That is let $T_{x}^{\text {in }} \mathcal{M}$ be the set of vectors $\xi \in T_{x} \mathcal{M}$ for which there exists a differentiable curve $\gamma:[0, \delta) \rightarrow \mathcal{M}$ such that $\gamma(0)=x$ and $\gamma^{\prime}(0)=\xi$. We note that the tangent space to $\partial M$, considered as a manifold, is a subset of the inward sector: $T \partial \mathcal{M} \subset T^{\text {in }} \mathcal{M}$.

The effort to infinitesimally move configuration $\mu$ in by a vector field $v \in T \mathcal{M}$ is

$$
\int_{\mathcal{M}} g_{x}(v(x), v(x)) d \mu(x)=\int_{\mathcal{M}} v^{T}(x) G(x) v(x) d \mu(x)
$$

where $G$ is the symmetric matrix which provides the metric $g$. However not all vector fields in $T \mathcal{M}$ are admissible as tangent vectors to a path in the configuration space. Namely the tangent vector fields must belong to the inward sector $T^{\text {in }} \mathcal{M}$. On the formal level, we consider admissible tangent vectors to the space of configurations to be vector fields in $T^{\text {in }} \mathcal{M}$ which are projections via $P$ of a continuous vector field in $T \mathcal{M}$. This is motivated by the fact, which we later establish, that gradient vector of energy $\mathcal{E}$ is given by $v=P w$ where $w$ is a continuous vector field $(w=$ $\left.\left(-G^{-1} \nabla(W * \mu+V)\right)\right)$.

The differential of $\mathcal{E}$ in the direction $v$ is given as the directional derivative

$$
\begin{aligned}
\operatorname{diff} \mathcal{E}[v] & =\left.\frac{d}{d t}\right|_{t=0} \mathcal{E}\left(\mu_{t}\right) \\
& =\left.\frac{d}{d t}\right|_{t=0}\left(\frac{1}{2} \int_{\mathcal{M}} \int_{\mathcal{M}} W\left(\Phi_{v}[t](x)-\Phi_{v}[t](y)\right) d \mu(x) d \mu(y)+\int_{\mathcal{M}} V\left(\Phi_{v}[t](x)\right) d \mu(x)\right) \\
& =\int_{\mathcal{M}}(\nabla W * \mu+\nabla V) v d \mu .
\end{aligned}
$$

Above we used that $\mu_{t}=\Phi_{v}[t]_{\sharp} \mu$ and the symmetry of $W$.

One can define the gradient descent of $\mathcal{E}$ with respect to metric given by $g$ by defining $-\operatorname{grad} \mathcal{E}$ to be the admissible vector field $v$ which minimizes

$$
\int_{\mathcal{M}} g(v, v) d \mu+\operatorname{diff} \mathcal{E}[v]
$$

that is

$$
\int_{\mathcal{M}} \frac{1}{2} v^{T} G v+\nabla(W * \mu+V) v d \mu
$$

To give this an interpretation of a true gradient flow we need to describe the tangent space to the space of configurations and endow it with an inner product. The issue is that more than one vector field can produce the same curve in the configuration space. Thus tangent vectors to the 
configuration space are defined as equivalence classes of admissible velocities which, for at least a short time, have the same flow map. The inner product of tangent vector fields is defined as

$$
\bar{g}(v, v)=\inf _{\tilde{v}}\left\{\int_{\mathcal{M}} \tilde{v}^{T} G \tilde{v} d \mu:(\exists \tilde{\delta}>0)(\forall t \in[0, \tilde{\delta})) \mu_{t}=\Phi_{\tilde{v}}[t]_{\sharp} \mu\right\} .
$$

The tangent vector field $v$ is considered as a representative of the class of velocities which produce the same curve. Since diff $E[v]$ does not depend on the representative tangent vector field chosen, we note that $-\operatorname{grad} \mathcal{E}$ we defined is also a minimizer of

$$
\frac{1}{2} \bar{g}(v, v)+\operatorname{diff} \mathcal{E}[v]
$$

over all tangent vectors $v$ at $\mu$; which agrees with the standard definition of a gradient flow on a manifold.

To determine the gradient vector we minimize the expression in (1.6). We obtain $v(x)=$ $-G^{-1} \nabla(W * \mu+V)(x)$ if $x$ is in the interior of $\mathcal{M}$ and also when $x \in \partial \mathcal{M}$ and $-G^{-1} \nabla(W * \mu+V)(x)$ is in the interior of $T_{x}^{\text {in }} \mathcal{M}$. Otherwise $v=\Pi_{\partial \mathcal{M}}\left(-G^{-1} \nabla(W * \mu+V)\right)$, where $\Pi_{\partial \mathcal{M}}$ is the orthogonal projection of $T_{x} \mathcal{M}$ to $T_{x} \partial \mathcal{M}$ with respect to $g$. Setting $A=G^{-1}$ and defining

$$
P \xi= \begin{cases}\xi, & \text { if } x \notin \partial \mathcal{M} \text { or } \xi \in T_{x}^{\text {in }} \mathcal{M} \\ \Pi_{\partial \mathcal{M}}(\xi), & \text { otherwise }\end{cases}
$$

gives that $-\operatorname{grad} \mathcal{E}$ is given by the vector field

$$
v=P(-A \nabla(W * \mu+V)) .
$$

The gradient flow of $\mathcal{E}$ is thus given by

$$
\frac{\partial}{\partial t} \mu+\operatorname{div}(\mu v)=0
$$

1.2. Setup and main results. We denote the usual Euclidean inner product by $\langle$,$\rangle . On manifold$ $(\mathcal{M}, g)$, for $\xi \in T_{x} \mathcal{M}$ we denote the norm associated to the metric $g$ as $|\xi|_{g}=\sqrt{g_{x}(\xi, \xi)}$. We denote the Euclidean gradient and Hessian by $\nabla$ and Hess and Riemannian gradient and Hessian by $\nabla_{\mathcal{M}}$ and Hess $\mathcal{M}$. For a function $f \in C^{0}(\mathcal{M})$, we say that $f$ is $\lambda$-geodesically convex on $(\mathcal{M}, g)$ if for any $x, y \in \mathcal{M}$ and any constant speed minimal geodesic $\gamma(t)$ connecting $x, y$ with $\gamma(0)=x, \gamma(1)=y$, we have

$$
f(\gamma(t)) \leq(1-t) f(x)+t f(y)-\frac{\lambda}{2} t(1-t) \operatorname{dist}^{2}(x, y) .
$$

Notice that if $f \in C^{2}(\mathcal{M})$ with $\operatorname{Hess}_{\mathcal{M}} f(x) \geq \lambda G(x)$ for all $x \in \mathcal{M}$, then $f$ is $\lambda$-geodesically convex on $(\mathcal{M}, g)$. We make the following assumptions on manifold $(\mathcal{M}, g)$ :

(M1) The Riemannian metric $g$ is $C^{2}$ and satisfies $|\xi|_{g}^{2} \geq \Lambda|\xi|^{2}$ for some constant $\Lambda$ and all $\xi \in T \mathcal{M}$

(M2) $(\mathcal{M}, g)$ is geodesically convex in that for all $x, y \in \mathcal{M}$ there exists a length minimizing geodesic contained in $\mathcal{M}$.

We also make the following assumptions on interaction potential $W$ and external potential $V$ :

(NL1) $W$ is continuous $W(0)=0$ and $W(x)=W(-x)$.

(NL2) $W(x, y):=W(x-y)$ is $\lambda$-geodesically convex on $(\mathcal{M} \times \mathcal{M}, g \times g)$ for some constant $\lambda$.

(NL3) $W \in C^{1}\left(\mathbb{R}^{d}\right)$ and $W(x-y) \leq C\left(1+\operatorname{dist}^{2}\left(x, x_{0}\right)+\operatorname{dist}^{2}\left(y, x_{0}\right)\right)$ for some $C>0$ and all $x, y \in \mathcal{M}$.

(NL4) $\liminf \operatorname{dist}\left((x, y),\left(x_{0}, x_{0}\right)\right) \rightarrow \infty \frac{W(x-y)}{\operatorname{dist}^{2}\left(x, x_{0}\right)+\operatorname{dist}^{2}\left(y, x_{0}\right)} \geq 0$.

(NL5) $V$ is $\lambda$-geodesically convex of $(\mathcal{M}, g)$.

(NL6) $V \in C^{1}(\mathcal{M})$ and $V(x) \leq C\left(1+\operatorname{dist}^{2}\left(x, x_{0}\right)\right)$ for all $x \in \mathcal{M}$.

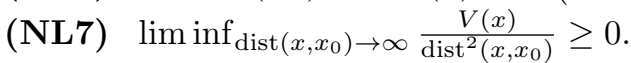


We list some remarks and simple consequences of the conditions. One can replace the condition (NL3) by the condition that $W \in C^{1}\left(\mathbb{R}^{d} \backslash\{0\}\right), W$ has local minimum at $x=0$ and satisfies the quadratic growth condition as in [13]. In this case $\partial^{\circ} \mathcal{E}=-P_{x}\left(-A(x)\left(\partial^{o} W * \mu+\nabla V\right)\right)$ where $\partial^{o} W * \mu(x)=\int_{y \neq x} \nabla W(x-y) d \mu(y)$ as defined in [13].

In (NL2) and (NL5), $W$ and $V$ may have different constants for convexity, but we assume that the constants are the same since we can take the minimum of the two constants if necessary.

Conditions (NL2) and (NL3) imply the following linear growth condition on $\nabla W$,

$$
\begin{aligned}
\left\langle A\left(x_{1}\right) \nabla W\left(x_{1}-y_{1}\right), \nabla W\left(x_{1}-y_{1}\right)\right\rangle & +\left\langle A\left(y_{1}\right) \nabla W\left(x_{1}-y_{1}\right), \nabla W\left(x_{1}-y_{1}\right)\right\rangle \\
\leq & C\left(1+\operatorname{dist}^{2}\left(x_{1}, x_{0}\right)+\operatorname{dist}^{2}\left(y_{1}, x_{0}\right)\right) .
\end{aligned}
$$

Similarly, (NL5) and (NL6) imply the linear growth condition on $\nabla V$,

$$
\langle A(x) \nabla V(x), \nabla V(x)\rangle \leq C\left(1+\operatorname{dist}^{2}\left(x, x_{0}\right)\right) .
$$

To see that, for $\nabla V$ we notice that

$$
\begin{aligned}
C\left(1+\operatorname{dist}^{2}\left(x, x_{0}\right)+\operatorname{dist}^{2}\left(y, x_{0}\right)\right) & \geq V(y)-V(x) \\
& \geq\langle\nabla V(x), T(x, y)\rangle+\frac{\lambda}{2} \operatorname{dist}^{2}(x, y),
\end{aligned}
$$

where $T(x, y)$ is the tangent vector at $x$ such that $\exp _{x}(T(x, y))=y$ and $|T(x, y)|_{g}=\operatorname{dist}(x, y)$, which we define in 3.1 in Section 3 . So

$$
C\left(1+\operatorname{dist}^{2}\left(x, x_{0}\right)+\operatorname{dist}^{2}(x, y)\right) \geq\langle\sqrt{A(x)} \nabla V(x), \sqrt{G(x)} T(x, y)\rangle+\frac{\lambda}{2} \operatorname{dist}^{2}(x, y) .
$$

Note that $\langle\sqrt{G(x)} T(x, y), \sqrt{G(x)} T(x, y)\rangle=\operatorname{dist}^{2}(x, y)$, by taking $\operatorname{dist}(x, y)=\max \left\{1, \operatorname{dist}\left(x, x_{0}\right)\right\}$, we get

$$
\langle A(x) \nabla V(x), \nabla V(x)\rangle \leq C\left(1+\operatorname{dist}^{2}\left(x, x_{0}\right)\right) .
$$

Similar calculations give the growth conditions on $\nabla W$.

Remark 1.1. (Simple conditions for (NL2) and (NL5)) In Section 6, we give detail calculations and precise conditions on $W, V$ and $g$ which guarantee $\lambda$-geodesic convexity of $V$ and $W$. Here we summarize some conclusions.

- If there exist constants $c_{1}>0, c_{2}>0$ such that the Riemannian metric $g \in C^{1}(\mathcal{M})$ with $c_{1} I_{d} \leq G(x) \leq \frac{1}{c_{1}} I_{d},\left|\frac{\partial}{\partial x_{k}} G_{i j}(x)\right| \leq \frac{1}{c_{1}}$ for all $x \in \mathcal{M}$ and $W$ is twice differentiable with $|\nabla W(y)| \leq c_{2}$, Hess $W(y) \geq-c_{2} I_{d}$ for all $y \in \mathcal{M}-\mathcal{M}:=\left\{x^{1}-x^{2}: x^{1}, x^{2} \in \mathcal{M}\right\}$, then $W$ is $\lambda$-geodesically convex on $(\mathcal{M} \times \mathcal{M}, g \times g)$.

- For $V$, if there exists a constant $c_{1}>0$ such that the Riemannian metric $g \in C^{1}(\mathcal{M})$ satisfies $c_{1} I_{d} \leq G(x) \leq \frac{1}{c_{1}} I_{d},\left|\frac{\partial}{\partial x_{k}} G_{i j}(x)\right| \leq \frac{1}{c_{1}}$ and $V \in C^{2}(\mathcal{M})$ satisfies that $|\nabla V(y)| \leq c_{2}$, Hess $V(y) \geq-c_{2} I_{d}$ for all $y \in \mathcal{M}$, then $V$ is $\lambda$-geodesically convex on $(\mathcal{M}, g)$.

- In general, the conditions on $g, W, V$ to guarantee $\lambda$-geodesic convexity of $W, V$ are more stringent than in the Euclidean space. For example: assuming $g \in C^{1}(\mathcal{M})$ such that $c_{1} I_{d} \leq G(x) \leq \frac{1}{c_{1}} I_{d},\left|\frac{\partial}{\partial x_{k}} G_{i j}\right| \leq \frac{1}{c_{1}}$ and Hess $V(y) \geq-c_{2} I_{d}$, Hess $W(y) \geq-c_{2} I_{d}$ for some constants $c_{1}>0, c_{2}>0$ does not imply $\lambda$-geodesic convexity of the energy. We present an explicit example in Section 6 (Example 6.3).

For manifolds satisfying (M1) and (M2) and potentials $W, V$ satisfying (NL1)-(NL7) we consider (1.9) as a gradient flow of $\mathcal{E}$ in space of probability measures endowed with the Riemannian Wasserstein metric. For the general theory on gradient flows in spaces of probability measures we refer to 
2. Denote the space of probability measures on $\mathcal{M}$ by $\mathcal{P}(\mathcal{M})$ and

$$
\mathcal{P}_{2}(\mathcal{M})=\left\{\mu \in \mathcal{P}(\mathcal{M}): \int_{\mathcal{M}} \operatorname{dist}^{2}\left(x, x_{0}\right) d \mu(x)<\infty\right\}
$$

for some $x_{0} \in \mathcal{M}$. Define the Riemannian 2-Wasserstein metric

$$
d_{W}^{2}(\nu, \mu)=\min \left\{\int_{\mathcal{M} \times \mathcal{M}} \operatorname{dist}^{2}(x, y) d \gamma(x, y): \gamma \in \Gamma(\mu, \nu)\right\}
$$

and the usual Euclidean 2-Wasserstein metric

$$
d_{W, E u c}^{2}(\nu, \mu)=\min \left\{\int_{\mathcal{M} \times \mathcal{M}}|x-y|^{2} d \gamma(x, y): \gamma \in \Gamma(\mu, \nu)\right\}
$$

where $\Gamma(\mu, \nu)$ is the set of joint probability distributions on $\mathcal{M} \times \mathcal{M}$ with first marginal $\mu$ and second marginal $\nu$, i.e.,

$$
\Gamma(\mu, \nu)=\left\{\gamma \in \mathcal{P}(\mathcal{M} \times \mathcal{M}):\left(\pi_{1}\right)_{\sharp} \gamma=\mu,\left(\pi_{2}\right)_{\sharp} \gamma=\nu\right\} .
$$

Denote the set of optimal transport plans between $\mu$ and $\nu$ with respect to the Riemannian 2Wasserstein metric $d_{W}$ by $\Gamma_{o}(\mu, \nu)$, that is

$$
\Gamma_{o}(\mu, \nu)=\left\{\gamma \in \Gamma(\mu, \nu): \int_{\mathcal{M} \times \mathcal{M}} \operatorname{dist}^{2}(x, y) d \gamma(x, y)=d_{W}^{2}(\mu, \nu)\right\} .
$$

We say that a functional $\mathcal{I}: \mathcal{P}_{2}(\mathcal{M}) \mapsto(-\infty, \infty]$ is $\lambda$-geodesically convex if for any $\mu, \nu \in \mathcal{P}_{2}(\mathcal{M})$ and any constant speed geodesic $\gamma$ with $\gamma(0)=\mu, \gamma(1)=\nu$, we have for any $0 \leq t \leq 1$,

$$
\mathcal{I}(\gamma(t)) \leq(1-t) \mathcal{I}(\mu)+t \mathcal{I}(\nu)-\frac{1}{2} \lambda t(1-t) d_{W}^{2}(\mu, \nu) .
$$

From calculations of Section 6, we know that if $W$ and $V$ are $\lambda$-geodesically convex on $(\mathcal{M} \times$ $\mathcal{M}, g \times g)$ and $(\mathcal{M}, g)$ respectively, then $\mathcal{E}$ is $\lambda$-geodesically convex on $\mathcal{P}_{2}(\mathcal{M})$, possibly with a different convexity constant. We now define the local slope of $\mathcal{E}$ with respect to the Riemannian 2-Wasserstein metric as

$$
|\partial \mathcal{E}|(\mu)=\limsup _{\nu \rightarrow \mu} \frac{(\mathcal{E}(\mu)-\mathcal{E}(\nu))^{+}}{d_{W}(\mu, \nu)},
$$

where $f^{+}=\max \{f, 0\}$ is the positive part of $f$. For a locally absolutely continuous curve $[0,+\infty) \ni$ $t \mapsto \mu(t) \in \mathcal{P}_{2}(\mathcal{M})$ with respect to Riemannian 2-Wasserstein metric $d_{W}$, we denote its metric derivative by

$$
\left|\mu^{\prime}\right|(t)=\limsup _{s \rightarrow t} \frac{d_{W}(\mu(t), \mu(s))}{|s-t|} .
$$

We call a locally absolutely continuous curve $[0,+\infty) \ni t \mapsto \mu(t) \in \mathcal{P}_{2}(\mathcal{M})$ a gradient flow with respect to the energy functional $\mathcal{E}$ if for a.e. $t>0$,

$$
v(t) \in-\partial \mathcal{E}(\mu(t))
$$

where $\partial \mathcal{E}(\mu(t))$ is the set of subdifferential of $\mathcal{E}$ at $\mu(t)$ and $v(t)$ is the tangent velocity of the curve at $\mu(t)$, which we define in Section 3 and Section 5 .

Define the weak measure solutions to the continuity equation by

Definition 1.2. A locally absolutely continuous curve $\mu(t) \in \mathcal{P}_{2}(\mathcal{M})$ is a weak measure solution to (1.9) with initial value $\mu_{0}$ if

$$
P\left(-A(x)\left(\int_{\mathcal{M}} \nabla W(x-y) d \mu(t, y)+\nabla V(x)\right)\right) \in L_{l o c}^{1}\left([0,+\infty) ; L^{2}(g, \mu(t))\right)
$$


and

$$
\begin{aligned}
\int_{0}^{\infty} \int_{\mathcal{M}} & \frac{\partial \phi}{\partial t}(t, x) d \mu(t, x) d t+\int_{\mathcal{M}} \phi(0, x) d \mu_{0}(x) \\
\quad & =-\int_{0}^{\infty} \int_{\mathcal{M}}\left\langle\nabla \phi(t, x), P\left(-A(x)\left(\int_{\mathcal{M}} \nabla W(x-y) d \mu(t, y)+\nabla V(x)\right)\right)\right\rangle d \mu(t, x) d t
\end{aligned}
$$

for all $\phi \in C_{c}^{\infty}([0, \infty) \times \mathcal{M})$.

Above we consider $C_{c}^{\infty}([0, \infty) \times \mathcal{M})$ to be the set of restrictions of functions in $C_{c}^{\infty}\left([0, \infty) \times \mathbb{R}^{d}\right)$ to $\mathcal{M}$. In particular we note that the values of test functions on the boundary of $\mathcal{M}$ may be different from zero. In this way the no-flux boundary conditions are imposed.

The main results of this paper are the following theorems regarding existence and stability of gradient flows with arbitrary initial data $\mu_{0} \in \mathcal{P}_{2}(\mathcal{M})$, which we prove in Section 5 .

Theorem 1.3. Assume (M1)-(M2) and (NL1)-(NL7), then for any $\mu_{0} \in \mathcal{P}_{2}(\mathcal{M})$ there exists a locally absolutely continuous curve $[0,+\infty) \ni t \mapsto \mu(t) \in \mathcal{P}_{2}(\mathcal{M})$ such that $\mu(0)=\mu_{0}$ and $\mu(t)$ is a gradient flow of $\mathcal{E}$ with respect to the Riemannian 2-Wasserstein metric $d_{W} \cdot \mu(t)$ satisfies that

$$
|\partial \mathcal{E}|^{2}(\mu(t))=\left|\mu^{\prime}\right|^{2}(t)=\int_{\mathcal{M}} g_{x}(\kappa(t, x), \kappa(t, x)) d \mu(t, x)
$$

and the energy dissipation equality, for $0 \leq s \leq t<\infty$

$$
\mathcal{E}(\mu(s))-\mathcal{E}(\mu(t))=\int_{s}^{t} \int_{\mathcal{M}} g_{x}(\kappa(r, x), \kappa(r, x)) d \mu(r, x) d r,
$$

where we denote $\kappa(r, x)=-P(-A(x)(\nabla W * \mu(r)(x)+\nabla V(x)))$. Moreover, $\mu(t)$ is a weak measure solution to (1.9) with initial data $\mu_{0}$.

Theorem 1.4. Suppose (M1)-(M2) and (NL1)-(NL7) hold true. Let $\mu^{1}(t), \mu^{2}(t)$ be two gradient flows of the energy functional $\mathcal{E}$ with initial data $\mu_{0}^{1}, \mu_{0}^{2}$ respectively, then

$$
d_{W}\left(\mu^{1}(t), \mu^{2}(t)\right) \leq e^{-\lambda t} d_{W}\left(\mu_{0}^{1}, \mu_{0}^{2}\right)
$$

for any $t \geq 0$. Moreover, the gradient flow solution is characterized by the system of Evolution Variational Inequalities:

$$
\frac{1}{2} \frac{d}{d t} d_{W}^{2}(\mu(t), \nu)+\frac{\lambda}{2} d_{W}^{2}(\mu(t), \nu) \leq \mathcal{E}(\nu)-\mathcal{E}(\mu(t)),
$$

for a.e. $t>0$ and for all $\nu \in \mathcal{P}_{2}(\mathcal{M})$.

\subsection{Remarks and connections.}

Remark 1.5. Recall that for interaction and potential energy on $\mathcal{P}_{2}\left(\mathbb{R}^{d}\right)$, the gradient flow of $\mathcal{E}$ with respect to the usual Euclidean 2-Wasserstein metric would be

$$
\frac{\partial}{\partial t} \mu(t, x)-\operatorname{div}\left(\mu(t, x)\left(\int_{\mathbb{R}^{d}} \nabla W(x-y) d \mu(t, y)+\nabla V(x)\right)\right)=0 .
$$

Comparing with 1.9 , we see that the projection $P_{x}$ is due to the boundary of $\mathcal{M}$ and the mobility $A$ comes from geometry of $\mathcal{M}$. is

We define the set of admissible vector fields $\mathfrak{V}$ at $\mu$ to be the set of $L^{2}(\mu)$ sections of $T^{\text {in }} \mathcal{M}$. That

$$
\mathfrak{V}=\left\{v: \mathcal{M} \rightarrow T \mathcal{M} \mid(\forall x \in \mathcal{M}) v(x) \in T_{x}^{\text {in }} \mathcal{M} \text { and } \int_{\mathcal{M}} g_{x}(v(x), v(x)) d \mu(x)<\infty\right\}
$$


Remark 1.6. If we assume that $\mathcal{M}$ has no boundary and we use the Riemannian volume form in defining the probability measures on $\mathcal{M}$, then the gradient flow of $\mathcal{E}$ with respect to the Riemannian 2 -Wasserstein metric is

$$
\frac{\partial}{\partial t} \mu(t, x)+\operatorname{div}_{\mathcal{M}}\left(\mu(t, x)\left(\int_{\mathcal{M}} \nabla_{\mathcal{M}} W(x-y) d \mu(t, y)+\nabla_{\mathcal{M}} V(x)\right)\right)=0,
$$

where the divergence and gradient should be understood as the Riemannian divergence and gradient on $\mathcal{M}$, and when test against test functions, it should be integrated against the Riemannian volume form $d \omega(x)$. Write (1.23) in local coordinates, we have

$$
\frac{\partial}{\partial t} \mu(t, x)+\frac{1}{\sqrt{\operatorname{det} G(x)}} \operatorname{div}\left(\mu(t, x) \sqrt{\operatorname{det} G(x)} A(x)\left(\int_{\mathcal{M}} \nabla W(x-y) d \mu(t, y)+\nabla V(x)\right)\right)=0,
$$

where the divergence is the Euclidean divergence now. We note that the equation above can be reduced to the form (1.9). Namely the measure $\tilde{\mu}$ defined by $d \tilde{\mu}(t, x)=\sqrt{\operatorname{det} G(x)} d \mu(t, x)$ solves

$$
\frac{\partial}{\partial t} \tilde{\mu}(t, x)+\operatorname{div}\left(\tilde{\mu}(t, x) A(x)\left(\int_{\mathcal{M}} \nabla W(x-y) d \tilde{\mu}(t, y)+\nabla V(x)\right)\right)=0,
$$

which is exactly 1.9 without the projection $P$. So it is similar to consider the gradient flow of $\mathcal{E}$ under the Riemannian and Euclidean volume form. Consequently, (NL1)-(NL7) also imply the existence of the gradient flow of $\mathcal{E}$ with respect to the Riemannian volume form.

1.4. Outline. In Section 2, we establish some important properties of the functional $\mathcal{E}$ and the manifold $\mathcal{M}$, in particular the lower semicontinuity of $\mathcal{E}$.

In Section 3, we give the definition of subdifferential in the manifold context, which is a natural generalization of the subdifferential in the Euclidean setting. We then identify the minimal subdifferential of $\mathcal{E}$ at $\mu$ as

$$
\partial^{o} \mathcal{E}(\mu)=-P\left(-A(x)\left(\int_{\mathcal{M}} \nabla W(x-y) d \mu(y)+\nabla V(x)\right)\right) .
$$

Section 4 is devoted to the JKO scheme. We show that the discrete scheme is well-posed and converges to a locally absolutely continuous curve $\mu(t) \in \mathcal{P}_{2}(\mathcal{M})$. Together with the fact the local slope $|\partial \mathcal{E}|$ is lower semicontinuous, we show that the limit curve $\mu(t)$ is a curve of maximal slope.

In Section 5 , we establish that the limit curve $\mu(t)$ we get from JKO scheme is actually a gradient flow, thus a weak measure solution to the continuity equation 1.9$)$. We then show that $\lambda$-geodesic convexity of the functional $\mathcal{E}$ implies uniqueness and stability of gradient flow solutions. We remark that the lack of existence of an appropriate flow map due to discontinuity of the velocity fields, makes the proof of differentiability of Wasserstein metric more involved (Lemma 5.3).

In Section 6, we give some examples of manifolds $(\mathcal{M}, g)$, external potentials $V$ and interaction potentials $W$ for which $V, W$ are $\lambda$-geodesically convex on $(\mathcal{M}, g)$ and $(\mathcal{M} \times \mathcal{M}, g \times g)$ respectively. These imply that functional $\mathcal{E}$ is $\lambda$-gedesically convex on $\left(\mathcal{P}_{2}(\mathcal{M}), d_{W}\right)$.

\section{Some properties of $\mathcal{E}$ AND $\mathcal{M}$}

In this section, we show some basic properties of the functionals $\mathcal{V}, \mathcal{W}$ and the manifold $\mathcal{M}$, which we need in the subsequent sections. First, we show the following simple relation between the distances of two points with respect to the Euclidean and Riemannian metric:

Lemma 2.1. For any $x, y \in \mathcal{M}$,

$$
\operatorname{dist}^{2}(x, y) \geq \Lambda|x-y|^{2} .
$$


Proof. Assume that $\gamma(t)$ is a curve which realizes the Riemannian distance between $x$ and $y$ and $\gamma(0)=x, \gamma(1)=y$, then we have

$$
\operatorname{dist}^{2}(x, y)=\int_{0}^{1} g_{\gamma(t)}\left(\gamma^{\prime}(t), \gamma^{\prime}(t)\right) d t \geq \int_{0}^{1} \Lambda\left\langle\gamma^{\prime}(t), \gamma^{\prime}(t)\right\rangle d t \geq \Lambda|x-y|^{2} .
$$

We can now compare the Wasserstein distance under Euclidean and Riemannian metric,

Lemma 2.2. For two Borel probability measures $\mu$ and $\nu$, we have

$$
d_{W}^{2}(\mu, \nu) \geq \Lambda d_{W, E u c}^{2}(\mu, \nu) .
$$

Proof. Assume that $\gamma$ is the optimal transportation plan between $\mu$ and $\nu$, that is $\gamma \in \Gamma_{o}(\mu, \nu)$. Then

$$
d_{W}^{2}(\mu, \nu)=\int_{\mathcal{M} \times \mathcal{M}} \operatorname{dist}(x, y)^{2} d \gamma(x, y) \geq \Lambda \int_{\mathcal{M} \times \mathcal{M}}|x-y|^{2} d \gamma(x, y) \geq \Lambda d_{W, E u c}^{2}(\mu, \nu) .
$$

Now we turn to the properties of $\mathcal{W}$ and $\mathcal{V}$.

Proposition 2.3 (Lower semicontinuity of $\mathcal{W}$ ). Assume (NL1)-(NL5), then

$$
\liminf _{n \rightarrow \infty} \mathcal{W}\left(\mu_{n}\right) \geq \mathcal{W}(\mu)
$$

given that $\mu_{n}$ narrowly converge to $\mu$ and $\mu_{n}$ have uniformly bounded second moments.

Proof. By (NL4), $\liminf _{\operatorname{dist}\left(x, x_{0}\right)+\operatorname{dist}\left(y, x_{0}\right) \rightarrow \infty} \frac{W(x-y)}{\operatorname{dist}^{2}\left(x, x_{0}\right)+\operatorname{dist}^{2}\left(y, x_{0}\right)} \geq 0$, for any $\varepsilon>0$, there exists $R>0$ such that

$$
\frac{W(x-y)}{\operatorname{dist}^{2}\left(x, x_{0}\right)+\operatorname{dist}^{2}\left(y, x_{0}\right)}>-\varepsilon
$$

for all $\operatorname{dist}\left(x, x_{0}\right)+\operatorname{dist}\left(y, x_{0}\right) \geq R$. Thus $W(x-y)+\varepsilon\left(\operatorname{dist}^{2}\left(x, x_{0}\right)+\operatorname{dist}^{2}\left(y, x_{0}\right)\right)$ is continuous and bounded from below. By Lemma 5.1.7 from [2], we know

$$
\begin{array}{r}
\liminf _{n \rightarrow \infty} \int_{\mathcal{M} \times \mathcal{M}}\left(W(x-y)+\varepsilon\left(\operatorname{dist}^{2}\left(x, x_{0}\right)+\operatorname{dist}^{2}\left(y, x_{0}\right)\right)\right) d \mu_{n}(x) d \mu_{n}(y) \\
\geq \int_{\mathcal{M} \times \mathcal{M}}\left(W(x-y)+\varepsilon\left(\operatorname{dist}^{2}\left(x, x_{0}\right)+\operatorname{dist}^{2}\left(y, x_{0}\right)\right)\right) d \mu(x) d \mu(y),
\end{array}
$$

which implies

$$
\begin{aligned}
\int_{\mathcal{M} \times \mathcal{M}} W(x-y) d \mu(x) d \mu(y) \leq & \liminf _{n \rightarrow \infty} \int_{\mathcal{M} \times \mathcal{M}} W(x-y) d \mu_{n}(x) d \mu_{n}(y) \\
& +\limsup _{n \rightarrow \infty} \int_{\mathcal{M} \times \mathcal{M}} \varepsilon\left(\operatorname{dist}^{2}\left(x, x_{0}\right)+\operatorname{dist}^{2}\left(y, x_{0}\right)\right) d \mu_{n}(x) d \mu_{n}(y)
\end{aligned}
$$

On the other hand,

$$
\int_{\mathcal{M} \times \mathcal{M}} \varepsilon\left(\operatorname{dist}^{2}\left(x, x_{0}\right)+\operatorname{dist}^{2}\left(y, x_{0}\right)\right) d \mu_{n}(x) d \mu_{n}(y) \leq 2 \varepsilon C
$$

where $C=\sup _{n} \int_{\mathcal{M}} \operatorname{dist}^{2}\left(x, x_{0}\right) d \mu_{n}(x)<\infty$. Taking $\varepsilon \rightarrow 0^{+}$yields

$$
\int_{\mathcal{M} \times \mathcal{M}} W(x-y) d \mu(x) d \mu(y) \leq \liminf _{n \rightarrow \infty} \int_{\mathcal{M} \times \mathcal{M}} W(x-y) d \mu_{n}(x) d \mu_{n}(y) .
$$


For $\mathcal{V}$ the following lower semicontinuity result holds:

Proposition 2.4 (Lower semicontinuity of $\mathcal{V})$. Assume (NL6)-(NL8), then

$$
\liminf _{n \rightarrow \infty} \mathcal{V}\left(\mu_{n}\right) \geq \mathcal{V}(\mu)
$$

given that $\mu_{n}$ narrowly converge to $\mu$ and $\mu_{n}$ have uniform bounded second moments.

The proof is analogous to the proof of lower semicontinuity of $\mathcal{W}$ and we omit it here.

We list some properties and observations about the projection $P$ :

- For any tangent vector field $v$ in $L^{2}(\mu), P v \in \mathfrak{V}$.

- In general for $v, w \in T_{x} \mathcal{M}, P\left(c_{1} v+c_{2} w\right) \neq c_{1} P v+c_{2} P w$ and $g(P v, w) \neq g(v, P w)$.

- For any $v, w \in T_{x} \mathcal{M},|P v-P w|_{g} \leq|v-w|_{g}$.

- $P$ can break the continuity of the velocity field. In particular if $\mu_{n}$ and $\mu$ are absolutely continuous curves in $\mathcal{P}_{2}(\mathcal{M})$ and $v_{n}$ and $v$ are corresponding velocities such that $\mu_{n}(t)$ converges narrowly to $\mu(t)$ then in the Euclidean setting (with no boundary) $v^{n} d \mu^{n}$ converges weakly to $v d \mu$, as was shown in Lemma 2.7 from [13. However this statement does not hold when boundary is present. Thus we need to use a different method to show the lower semicontinuity of the local slope $|\partial \mathcal{E}|$, which we do that in Theorem 4.4

- Even though $P$ is non-linear and breaks continuity, we still have that: The function $\mathcal{M} \times$ $\mathbb{R}^{d} \ni(x, \xi) \mapsto g_{x}\left(P_{x} \xi, P_{x} \xi\right)$ is lower semicontinuous and for all $x \in \mathcal{M}$, the function $\mathbb{R}^{d} \ni \xi \mapsto g_{x}\left(P_{x} \xi, P_{x} \xi\right)$ is convex. Refer to Proposition 4.6 for the proof.

\section{Minimal subdifferential of $\mathcal{E}$}

In this section, we give the definition of subdifferetial in the Riemannian geometric setting, which is the natural generalization of the notion in the Euclidean setting. We then identify the minimal subdifferential of $\mathcal{E}$ as $\partial^{\circ} \mathcal{E}(\mu)=-P(-A(\nabla W * \mu+\nabla V))$ and show that it realizes the local slope in the sense that $|\partial \mathcal{E}|(\mu)=\left\|\partial^{o} \mathcal{E}(\mu)\right\|_{L^{2}(g, \mu)}$.

In order to define the subdifferential of a functional in the Riemannian setting, we introduce the exponential map on the space of configurations first. Let $\exp _{x}: T_{x} \mathcal{M} \rightarrow \mathcal{M}$ be the exponential map on $\mathcal{M}$. It is understood that the domain of $\exp _{x}$ is actually a subset of $T_{x} \mathcal{M}$ for which the geodesics of appropriate length and direction exists. We note that if $x$ is in the interior of $\mathcal{M}$ then the domain of $\exp _{x}$ is an open neighborhood of 0 , while if $x \in \partial \mathcal{M}$ then the domain of $\exp _{x}$ a subset of $T_{x}^{\text {in }} \mathcal{M}$ and may not be an open neighborhood of 0 even in $T_{x}^{\text {in }} \mathcal{M}$. For example if $\mathcal{M}=B(0,1)$, $g$ is the Euclidean metric, $x=(1,0)$, and $\xi=(0,1)$, then $\xi \in T_{x}^{\text {in }} \mathcal{M}$, but $\exp (t \xi)$ is not defined for any $t \neq 0$. This required us to modify a number of standard arguments so that we do not use the exponential map to generate geodesics. We only use the exponential map to parameterize the geodesics which we know to exist.

By our assumptions on $(\mathcal{M}, g)$ we know that there exists a length minimizing geodesics connecting any two points. The problem is that such geodesics may not be unique. However, by Aumann measurable selection theorem, see 25, geodesics can be selected in a measurable way. More precisely there exists a measurable function $T: \mathcal{M} \times \mathcal{M} \rightarrow T^{\text {in }} \mathcal{M}$ such that for all $x, y \in \mathcal{M}$

$$
\exp _{x}(T(x, y))=y
$$

and such that $\gamma(t)=\exp _{x}(t T(x, y)), t \in[0,1]$ gives a minimal geodesic connecting $x$ and $y$. Note that $g_{x}(T(x, y), T(x, y))=\operatorname{dist}^{2}(x, y)$. Unless otherwise specified, in the remainder of the paper, by $T$ we denote an arbitrary measurable function satisfying the above.

Definition 3.1 (Subdifferential). Fix $\mu \in \mathcal{P}_{2}(\mathcal{M})$, a vector field $\xi \in L^{2}(g, \mu)$ is said to be an element of the subdifferential of $\mathcal{E}$ at $\mu$, and we denote as $\xi \in \partial \mathcal{E}(\mu)$, if there exists $T: \mathcal{M} \times \mathcal{M} \rightarrow T \mathcal{M}$ as 
in (3.1) such that

$$
\mathcal{E}(\nu)-\mathcal{E}(\mu) \geq \inf _{\gamma \in \Gamma_{o}(\mu, \nu)} \int_{\mathcal{M} \times \mathcal{M}} g_{x}(\xi(x), T(x, y)) d \gamma(x, y)+o\left(d_{W}(\mu, \nu)\right),
$$

where $\Gamma_{o}(\mu, \nu)$ is the optimal plan between $\mu$ and $\nu$ as defined in 1.13 .

We denote the element in $\partial \mathcal{E}(\mu)$ with minimal $L^{2}(g, \mu)$ norm by $\partial^{o} \mathcal{E}(\mu)$.

Remark 3.2. Notice that Definition 3.1 reduces to the usual definition of subdifferential when $g$ is the Euclidean metric. It is straightforward calculation to show that if $\xi \in \partial \mathcal{E}(\mu)$ then

$$
|\partial \mathcal{E}|(\mu) \leq\|\xi\|_{L^{2}(g, \mu)},
$$

where $\|\xi\|_{L^{2}(g, \mu)}^{2}=\int_{\mathcal{M}} g_{x}(\xi(x), \xi(x)) d \mu(x)$.

We now give the following main theorem of this section regarding the existence of subdifferential and the minimal $L^{2}(g, \mu)$ element of the subdifferential.

Theorem 3.3. Assume (M1)-(M2), (NL1)-(NL7) hold, then $\partial \mathcal{E}(\mu) \neq \emptyset$ for any $\mu \in \mathcal{P}_{2}(\mathcal{M})$. Moreover the vector field

$$
\kappa(x)=-P_{x}\left(-A(x)\left(\int_{\mathcal{M}} \nabla W(x-y) d \mu(y)+\nabla V(x)\right)\right)
$$

is the unique element of minimal $L^{2}(g, \mu)$-norm in $\partial \mathcal{E}(\mu)$ with

$$
|\partial \mathcal{E}|(\mu)=\|\kappa\|_{L^{2}(g, \mu)} .
$$

Remark 3.4. To consider interaction potentials $W \in C^{1}\left(\mathbb{R}^{d} \backslash\{0\}\right)$, one needs to notice that $0 \in \partial W(0)$. The proof of the above theorem can be used to show that the minimal subdifferential is

$$
\partial^{o} \mathcal{E}(\mu)=-P\left(-A\left(\partial^{o} W * \mu+\nabla V\right)\right),
$$

where $\partial^{\circ} W(x)=\nabla W(x)$ if $x \neq 0$ and $\partial^{\circ} W(0)=0$.

We also remark that while in the definition of subdifferential Definition 3.1, we only require (3.2) to hold for some measurable choice of $T(x, y)$ and infimum over $\gamma \in \Gamma_{o}(\mu, \nu)$, in the proof we actually show that for any $\gamma \in \Gamma_{o}(\mu, \nu)$ and any measurable selection $T(x, y), 3.2$ holds true with that particular choice of $T(x, y)$ and $\gamma$.

Before proving the theorem, we need the following

Lemma 3.5. Let $\xi$ be a vector field in $\mathfrak{V}$ such that there exists $t_{0}>0$ for which $\exp _{x}(t \xi(x)) \in \mathcal{M}$ for all $0 \leq t \leq t_{0}$ and $x \in \mathcal{M}$. Then

$$
\limsup _{t \rightarrow 0^{+}} \frac{d_{W}\left((\exp (t \xi))_{\sharp} \mu, \mu\right)}{t} \leq\|\xi\|_{L^{2}(g, \mu)},
$$

where we denote $\exp (t \xi)(x)=\exp _{x}(t \xi(x))$.

Proof of Lemma. For $0 \leq t<t_{0}$, notice that $(i d, \exp (t \xi))_{\sharp} \mu \in \Gamma\left(\mu,(\exp (t \xi))_{\sharp} \mu\right)$, so

$$
\begin{aligned}
d_{W}^{2}\left(\mu,(\exp (t \xi))_{\sharp} \mu\right) & \leq \int_{\mathcal{M}} \operatorname{dist}^{2}\left(x, \exp _{x}(t \xi(x))\right) d \mu(x) \\
& \leq \int_{\mathcal{M}} t^{2} g_{x}(\xi(x), \xi(x)) d \mu(x) .
\end{aligned}
$$


Thus

$$
\limsup _{t \rightarrow 0^{+}} \frac{d_{W}^{2}\left(\mu,(\exp (t \xi))_{\sharp} \mu\right)}{t^{2}} \leq \int_{\mathcal{M}} g_{x}(\xi(x), \xi(x)) d \mu(x) .
$$

We now prove the theorem.

Proof of Theorem. We divide the proof into two steps.

Step 1. $\kappa \in \partial \mathcal{E}(\mu)$. We need to prove that

$$
\int_{\mathcal{M}} g_{x}(\kappa(x), \kappa(x)) d \mu(x)<\infty
$$

and

$$
\mathcal{E}(\nu)-\mathcal{E}(\mu) \geq \inf _{\gamma \in \Gamma_{o}(\mu, \nu)} \int_{\mathcal{M} \times \mathcal{M}} g_{x}(\kappa(x), T(x, y)) d \gamma(x, y)+o\left(d_{W}(\mu, \nu)\right) .
$$

To prove the first claim, note that

$$
\begin{aligned}
& \int_{\mathcal{M}} g(\kappa(x), \kappa(x)) d \mu(x) \\
& \leq \int_{\mathcal{M}} g\left(A(x)\left(\int_{\mathcal{M}} \nabla W(x-y) d \mu(y)+\nabla V(x)\right), A(x)\left(\int_{\mathcal{M}} \nabla W(x-y) d \mu(y)+\nabla V(x)\right)\right) d \mu(x) \\
& =\int_{\mathcal{M}}\left\langle A(x)\left(\int_{\mathcal{M}} \nabla W(x-y) d \mu(y)+\nabla V(x)\right), \int_{\mathcal{M}} \nabla W(x-y) d \mu(y)+\nabla V(x)\right\rangle d \mu(x) \\
& \leq \int_{\mathcal{M} \times \mathcal{M}}\langle A(x)(\nabla W(x-y)+\nabla V(x)), \nabla W(x-y)+\nabla V(x)\rangle d \mu(y) d \mu(x) \\
& \leq \int_{\mathcal{M} \times \mathcal{M}} C\left(1+\operatorname{dist}^{2}\left(x, x_{0}\right)+\operatorname{dist}^{2}\left(y, x_{0}\right)\right) d \mu(x) d \mu(y) \\
& <\infty .
\end{aligned}
$$

The first inequality above comes from the fact that projection does not increase the length of a vector, while the third inequality holds because $\nabla W$ and $\nabla V$ have liner growth, as shown in 1.10 and (1.11). The last inequality holds since $\mu$ has finite second moment.

To prove the second claim let $\mu, \nu \in \mathcal{P}_{2}(\mathcal{M}), \gamma \in \Gamma_{o}(\mu, \nu)$ be any optimal plan and $T(x, y)$ be as in (3.1). Due to $\lambda$-convexity of $W$ and $V$, the function

$$
\begin{aligned}
f(t)= & \frac{W\left(\exp _{x_{1}}\left(t T\left(x_{1}, y_{1}\right)\right)-\exp _{x_{2}}\left(t T\left(x_{2}, y_{2}\right)\right)\right)-W\left(x_{1}-x_{2}\right)}{2 t} \\
& +\frac{2 V\left(\exp _{x_{2}}\left(t T\left(x_{2}, y_{2}\right)\right)\right)-2 V\left(x_{2}\right)}{2 t}-\frac{\lambda}{2} t \operatorname{dist}^{2}\left(x_{2}, y_{2}\right)-\frac{\lambda}{2} t \operatorname{dist}^{2}\left(\left(x_{1}, x_{2}\right),\left(y_{1}, y_{2}\right)\right)
\end{aligned}
$$

is non-decreasing on $[0,1]$, so $f(1) \geq \liminf _{t \rightarrow 0^{+}} f(t)$. We remark here that the fact that the curve $t \mapsto \exp _{x_{1}}\left(t T\left(x_{1}, y_{1}\right)\right)-\exp _{x_{2}}\left(t T\left(x_{2}, y_{2}\right)\right)$ is no longer a geodesic on $(\mathcal{M}, g)$ is the reason why we need to assume (NL2) of $W$, i.e. the $\lambda$-geodesic convexity of $(x, y) \in \mathcal{M} \times \mathcal{M} \mapsto W(x, y)=W(x-y)$ instead of $\lambda$-geodesic convexity of $x \in \mathcal{M} \mapsto W(x)$ as in the Euclidean setting. Note that

$$
\begin{aligned}
& \lim _{t \rightarrow 0^{+}}\left[\frac{W\left(\exp _{x_{1}}\left(t T\left(x_{1}, y_{1}\right)\right)-\exp _{x_{2}}\left(t T\left(x_{2}, y_{2}\right)\right)\right)-W\left(x_{1}-x_{2}\right)}{2 t}-\frac{\lambda}{2} t \operatorname{dist}^{2}\left(\left(x_{1}, x_{2}\right),\left(y_{1}, y_{2}\right)\right)\right] \\
& =\frac{1}{2}\left\langle\nabla W\left(x_{1}-x_{2}\right), T\left(x_{1}, y_{1}\right)-T\left(x_{2}, y_{2}\right)\right\rangle,
\end{aligned}
$$


and

$$
\lim _{t \rightarrow 0^{+}}\left[\frac{V\left(\exp _{x_{2}}\left(t T\left(x_{2}, y_{2}\right)\right)\right)-V\left(x_{2}\right)}{t}-\frac{\lambda}{2} t \operatorname{dist}^{2}\left(x_{2}, y_{2}\right)\right]=\left\langle\nabla V\left(x_{2}\right), T\left(x_{2}, y_{2}\right)\right\rangle
$$

Then integrating over $d \gamma\left(x_{1}, y_{1}\right) d \gamma\left(x_{2}, y_{2}\right)$ gives

$$
\begin{aligned}
\mathcal{E}(\nu) & -\mathcal{E}(\mu)=\int_{\mathcal{M} \times \mathcal{M}} \int_{\mathcal{M} \times \mathcal{M}} \frac{W\left(y_{1}-y_{2}\right)+2 V\left(y_{2}\right)-W\left(x_{1}-x_{2}\right)-2 V\left(x_{2}\right)}{2} d \gamma\left(x_{1}, y_{1}\right) d \gamma\left(x_{2}, y_{2}\right) \\
\geq & \int_{\mathcal{M} \times \mathcal{M}} \int_{\mathcal{M} \times \mathcal{M}}\left[\frac{1}{2}\left\langle\nabla W\left(x_{1}-x_{2}\right), T\left(x_{1}, y_{1}\right)-T\left(x_{2}, y_{2}\right)\right\rangle+\left\langle\nabla V\left(x_{2}\right), T\left(x_{2}, y_{2}\right)\right\rangle\right] d \gamma\left(x_{1}, y_{1}\right) d \gamma\left(x_{2}, y_{2}\right) \\
& +o\left(d_{W}(\mu, \nu)\right) \\
= & \int_{\mathcal{M} \times \mathcal{M}} \int_{\mathcal{M} \times \mathcal{M}}\left\langle\nabla W\left(x_{2}-x_{1}\right)+\nabla V\left(x_{2}\right), T\left(x_{2}, y_{2}\right)\right\rangle d \gamma\left(x_{1}, y_{1}\right) d \gamma\left(x_{2}, y_{2}\right)+o\left(d_{W}(\mu, \nu)\right) \\
= & \int_{\mathcal{M} \times \mathcal{M}}\left\langle\int_{\mathcal{M}} \nabla W\left(x_{2}-x_{1}\right) d \mu\left(x_{1}\right)+\nabla V\left(x_{2}\right), T\left(x_{2}, y_{2}\right)\right\rangle d \gamma\left(x_{2}, y_{2}\right)+o\left(d_{W}(\mu, \nu)\right) \\
= & \int_{\mathcal{M} \times \mathcal{M}} g_{x_{2}}\left(A\left(x_{2}\right)\left(\int_{\mathcal{M}} \nabla W\left(x_{2}-x_{1}\right) d \mu\left(x_{1}\right)+\nabla V\left(x_{2}\right)\right), T\left(x_{2}, y_{2}\right)\right) d \gamma\left(x_{2}, y_{2}\right)+o\left(d_{W}(\mu, \nu)\right) \\
\geq & -\int_{\mathcal{M} \times \mathcal{M}} g_{x_{2}}\left(P_{x_{2}}\left(-A\left(x_{2}\right)\left(\int_{\mathcal{M}} \nabla W\left(x_{2}-x_{1}\right) d \mu\left(x_{1}\right)+\nabla V\left(x_{2}\right)\right)\right), T\left(x_{2}, y_{2}\right)\right) d \gamma\left(x_{2}, y_{2}\right) \\
& +o\left(d_{W}(\mu, \nu)\right) \\
= & \int_{\mathcal{M} \times \mathcal{M}} g_{x_{2}}\left(\kappa\left(x_{2}\right), T\left(x_{2}, y_{2}\right)\right) d \gamma\left(x_{2}, y_{2}\right)+o\left(d_{W}(\mu, \nu)\right)
\end{aligned}
$$

where the second inequality comes from the fact that: If $x_{2} \notin \partial \mathcal{M}$, by definition of $P_{x_{2}}$ the inequality becomes an equality while if $x_{2} \in \partial \mathcal{M}$, then by definition of $P_{x_{2}}$

$$
\begin{aligned}
& g_{x_{2}}\left(A\left(x_{2}\right)\left(\int_{\mathcal{M}} \nabla W\left(x_{2}-x_{1}\right) d \mu\left(x_{1}\right)+\nabla V\left(x_{2}\right)\right), \xi\right) \\
\geq & g_{x_{2}}\left(-P_{x_{2}}\left(-A\left(x_{2}\right)\left(\int_{\mathcal{M}} \nabla W\left(x_{2}-x_{1}\right) d \mu\left(x_{1}\right)+\nabla V\left(x_{2}\right)\right)\right), \xi\right)
\end{aligned}
$$

for any $\xi \in T_{x_{2}}^{\text {in }} M$, and we notice that $T\left(x_{2}, y_{2}\right) \in T_{x_{2}}^{\text {in }} M$.

Step 2. $\kappa$ is the element of minimal $L^{2}(g, \mu)$-norm in $\partial \mathcal{E}(\mu)$. By Remark 3.2 , we only need to show $\|\kappa\|_{L^{2}(g, \mu)} \leq|\partial \mathcal{E}|(\mu)$. Consider first a vector field $\xi$ as in Lemma 3.5 , i.e. $\xi \in L^{2}(g, \mu)$ and 
$\exp _{x}(t \xi(x)) \in \mathcal{M}$ for all $x \in \mathcal{M}$ and $0 \leq t \leq t_{0}$,

$$
\begin{aligned}
& \lim _{t \rightarrow 0^{+}} \frac{\mathcal{E}\left(\exp (t \xi)_{\sharp} \mu\right)-\mathcal{E}(\mu)}{t} \\
= & \lim _{t \rightarrow 0^{+}} \frac{1}{2} \int_{\mathcal{M} \times \mathcal{M}} \frac{W\left(\exp _{x}(t \xi(x))-\exp _{z}(t \xi(z))\right)+2 V\left(\exp _{x}(t \xi(x))\right)-W(x-z)-2 V(x)}{t} d \mu(x) d \mu(z) \\
= & \frac{1}{2} \int_{\mathcal{M} \times \mathcal{M}}\langle\nabla W(x-z), \xi(x)-\xi(z)\rangle+2\langle\nabla V(x), \xi(x)\rangle d \mu(x) d \mu(z) \\
= & \int_{\mathcal{M}}\left\langle\int_{\mathcal{M}} \nabla W(x-z) d \mu(z)+\nabla V(x), \xi(x)\right\rangle d \mu(x) \\
= & \int_{\mathcal{M}}\left\langle A(x)\left(\int_{\mathcal{M}} \nabla W(x-z) d \mu(z)+\nabla V(x)\right), G(x) \xi(x)\right\rangle d \mu(x) \\
= & \int_{\mathcal{M}} g_{x}\left(A(x)\left(\int_{\mathcal{M}} \nabla W(x-y) d \mu(y)+\nabla V(x)\right), \xi(x)\right) d \mu(x)
\end{aligned}
$$

given that we can prove the second equality. By the $\lambda$-convexity of $W$,

$$
\begin{aligned}
& t \nabla W(x-z) \cdot(\xi(x)-\xi(z))+\frac{\lambda}{2} t^{2}(g(\xi(x), \xi(x))+g(\xi(z), \xi(z))) \\
& \leq W\left(\exp _{x}(t \xi(x))-\exp _{z}(t \xi(z))\right)-W(x-z) \\
& \leq \nabla W\left(\exp _{x}(t \xi(x))-\exp _{z}(t \xi(z))\right) t \frac{d}{d t}\left(\exp _{x}(t \xi(x))-\exp _{z}(t \xi(z))\right) \\
& -\frac{\lambda}{2} t^{2}(g(\xi(x), \xi(x))+g(\xi(z), \xi(z))) .
\end{aligned}
$$

Then by linear growth condition on $\nabla W(1.10)$, we know that

$$
\begin{aligned}
& \left|t \nabla W(x-z) \cdot(\xi(x)-\xi(z))+\frac{\lambda}{2} t^{2}(g(\xi(x), \xi(x))+g(\xi(z), \xi(z)))\right| \\
& \leq C\left(1+\operatorname{dist}\left(x, x_{0}\right)+\operatorname{dist}\left(z, x_{0}\right)\right) t\left(g(\xi(x), \xi(x))^{\frac{1}{2}}+g(\xi(z), \xi(z))^{\frac{1}{2}}\right)+|\lambda| t^{2}(g(\xi(x), \xi(x))+g(\xi(z), \xi(z))) .
\end{aligned}
$$

Similarly

$$
\begin{aligned}
& \mid \nabla W\left(\exp _{x}(t \xi(x))-\exp _{z}(t \xi(z))\right) t \frac{d}{d t}\left(\exp _{x}(t \xi(x))-\exp _{z}(t \xi(z))\right) \\
& -\frac{\lambda}{2} t^{2}(g(\xi(x), \xi(x))+g(\xi(z), \xi(z))) \mid \\
\leq & C\left(1+\operatorname{dist}\left(\exp _{x}(t \xi(x)), x_{0}\right)+\operatorname{dist}\left(\exp _{z}(t \xi(z)), x_{0}\right)\right) t\left(g(\xi(x), \xi(x))^{\frac{1}{2}}+g(\xi(z), \xi(z))^{\frac{1}{2}}\right) \\
& +|\lambda| t^{2}(g(\xi(x), \xi(x))+g(\xi(z), \xi(z))) \\
\leq & C\left(1+\operatorname{dist}\left(x, x_{0}\right)+\operatorname{dist}\left(z, x_{0}\right)+t g(\xi(x), \xi(x))^{\frac{1}{2}}+\operatorname{tg}(\xi(z), \xi(z))^{\frac{1}{2}}\right) t\left(g(\xi(x), \xi(x))^{\frac{1}{2}}+g(\xi(z), \xi(z))^{\frac{1}{2}}\right) \\
& +|\lambda| t^{2}(g(\xi(x), \xi(x))+g(\xi(z), \xi(z))) .
\end{aligned}
$$

Then for $V$,

$$
\begin{aligned}
& (\nabla V(x), t \xi(x))+\lambda t^{2} g(\xi(x), \xi(x)) \\
& \leq V\left(\exp _{x}(t \xi(x))\right)-V(x) \\
& \leq \nabla V\left(\exp _{x}(t \xi(x))\right) t \frac{d}{d t} \exp _{x}(t \xi(x))-\lambda t^{2} g(\xi(x), \xi(x))
\end{aligned}
$$


By similar arguments, we have

$$
\left|(\nabla V(x), t \xi(x))+\lambda t^{2} g(\xi(x), \xi(x))\right| \leq C\left(1+\operatorname{dist}\left(x, x_{0}\right)\right) t g(\xi(x), \xi(x))^{\frac{1}{2}}+|\lambda| t^{2} g(\xi(x), \xi(x))
$$

and

$$
\begin{aligned}
& \left|\nabla V\left(\exp _{x}(t \xi(x))\right) t \frac{d}{d t} \exp _{x}(t \xi(x))-\lambda t^{2} g(\xi(x), \xi(x))\right| \\
& \leq\left(1+\operatorname{dist}\left(x, x_{0}\right)+t g(\xi(x), \xi(x))^{\frac{1}{2}}\right) \operatorname{tg}(\xi(x), \xi(x))^{\frac{1}{2}}+|\lambda| t^{2} g(\xi(x), \xi(x)) .
\end{aligned}
$$

Dividing by $t$ and noting that $\int_{\mathcal{M}} g_{x}(\xi(x), \xi(x)) d \mu(x)<\infty$ and $\int_{\mathcal{M}} \operatorname{dist}^{2}\left(x, x_{0}\right) d \mu(x)<\infty$ gives the second equality by Lebesgue's dominated convergence theorem.

By the definition of local slope 1.14 and Lemma 3.5

$$
\begin{aligned}
|\partial \mathcal{E}|(\mu)\|\xi\|_{L^{2}(g, \mu)} & \geq|\partial \mathcal{E}|(\mu) \liminf _{t \rightarrow 0^{+}} \frac{\left.d_{W}(\exp (t \xi))_{\sharp} \mu, \mu\right)}{t} \\
& \geq-\int_{\mathcal{M}}\left\langle A(x)\left(\int_{\mathcal{M}} \nabla W(x-z) d \mu(z)+\nabla V(x)\right), G(x) \xi(x)\right\rangle d \mu(x) \\
& =\int_{\mathcal{M}} g_{x}\left(-A(x)\left(\int_{\mathcal{M}} \nabla W(x-z) d \mu(z)+\nabla V(x)\right), \xi(x)\right) d \mu(x) .
\end{aligned}
$$

We need to plug $\xi=-\kappa$ into $(3.9)$, however it is possible that there exists $x \in \partial \mathcal{M}$, such that there exists no $t_{0}>0$ with $\exp _{x}(-t \kappa(x)) \in \mathcal{M}$ for all $0 \leq t \leq t_{0}$. Thus we perform the following approximation scheme. For $n \in \mathbb{N}$, denote $\mathcal{M}_{n}=\left\{x \in \mathcal{M}: \operatorname{dist}(x, \partial \mathcal{M})>\frac{1}{n}\right\}, B(n)=\{x \in \mathcal{M}$ : $\left.\operatorname{dist}\left(x, x_{0}\right)<n\right\}$ and $n(x)$ the outward normal direction with respect to the Rimmannian metric at $x \in \partial \mathcal{M}$. Define

$$
\xi_{n}(x)= \begin{cases}-\kappa(x) & \text { if } x \in B_{n} \cap \mathcal{M}_{n}, \\ -\kappa(x)-\frac{1}{n} n(x) & \text { if } x \in B_{n} \cap \partial \mathcal{M}, \\ 0 & \text { Otherwise }\end{cases}
$$

We claim that $\xi_{n}$ satisfies the conditions in Lemma 3.5 and $\xi_{n}$ converges to $-\kappa$ in $L^{2}(g, \mu)$. Indeed, it is straightforward to see that $\xi_{n} \in L^{2}(g, \mu)$ and $\xi_{n}$ converges to $-\kappa$ in $L^{2}(g, \mu)$. Since $\kappa$ is continuous in $\mathcal{M}_{n}$ and $B(n) \Subset \mathcal{M}$, we have $\|\kappa\|_{L^{\infty}(g, \mu)} \leq C(n)$ on $B(n) \cap \mathcal{M}_{n}$ and thus for $0 \leq t \leq \frac{1}{n C(n)}$, $\exp _{x}\left(t \xi_{n}(x)\right) \in \mathcal{M}$ for $x \in B(n) \cap \mathcal{M}_{n}$. For $x \in \overline{B(n) \cap \partial \mathcal{M}}$, we know $g_{x}\left(\xi_{n}(x), n(x)\right) \leq-\frac{1}{n}$ and $\overline{B(n) \cap \partial \mathcal{M}}$ is compact, so there exists $\tilde{t}(n)$ such that $\exp _{x}\left(t \xi_{n}(x)\right) \in \mathcal{M}$ for all $0 \leq t \leq \tilde{t}(n)$ and $x \in \overline{B(n) \cap \partial \mathcal{M}}$. We can take $t_{0}=\min \left\{\frac{1}{n C(n)}, \tilde{t}(n)\right\}$ and $\exp _{x}\left(t \xi_{n}(x)\right) \in \mathcal{M}$ for $0 \leq t \leq t_{0}$ as claimed. Using $\xi_{n}$ in $(3.9)$ yields

$$
|\partial \mathcal{E}|(\mu)\left\|\xi_{n}\right\|_{L^{2}(g, \mu)} \geq \int_{\mathcal{M}} g_{x}\left(-A(x)\left(\int_{\mathcal{M}} \nabla W(x-z) d \mu(z)+\nabla V(x)\right), \xi_{n}(x)\right) d \mu(x) .
$$

Since $g_{x}(\xi(x), P \xi(x))=g_{x}(P \xi(x), P \xi(x))$, taking $n \rightarrow \infty$ then gives

$$
\begin{aligned}
|\partial \mathcal{E}|(\mu)\|\kappa\|_{L^{2}(g, \mu)} & \geq \int_{\mathcal{M}} g_{x}\left(-A(x)\left(\int_{\mathcal{M}} \nabla W(x-z) d \mu(z)+\nabla V(x)\right),-\kappa(x)\right) d \mu(x) \\
& =\int_{\mathcal{M}} g_{x}(\kappa(x), \kappa(x)) d \mu(x) .
\end{aligned}
$$

Hence

$$
\|\kappa\|_{L^{2}(g, \mu)} \leq|\partial \mathcal{E}|(\mu)
$$

which completes the proof. 


\section{JKO SCHEME: EXISTENCE OF MINIMIZERS AND CONVERGENCE}

In this section, we give the definition of curves of maximal slope and show their existence. The general framework, developed in [2], uses the JKO scheme, which we describe below. We verify the conditions on the functional $\mathcal{E}$ needed to apply the general existence theorem of [2] to get a curve of maximal slope with respect to the relaxed local slope $\left|\partial^{-} \mathcal{E}\right|$. In order to show that the limit curve is a curve of maximal slope with respect to $|\partial \mathcal{E}|$, we proceed to prove that $\mathcal{P}_{2}(\mathcal{M}) \ni \mu \mapsto \int_{s}^{t}|\partial \mathcal{E}|^{2}(\mu(r)) d r$ is lower semicontinuous with respect to narrow convergence of probability measures.

We start with the definition of curves of maximal slope.

Definition 4.1. A locally absolutely continuous curve $[0, \infty) \ni t \mapsto \mu(t) \in \mathcal{P}_{2}(\mathcal{M})$ is a curve of maximal slope for the functional $\mathcal{E}$ with respect to upper gradient $g$, if $\mathcal{E} \circ \mu$ is $\mathscr{L}^{1}$-a.e. equal to a non-increasing function $\varphi$ and

$$
\varphi^{\prime}(t) \leq-\frac{1}{2}\left|\mu^{\prime}\right|^{2}(t)-\frac{1}{2} g^{2}(\mu(t))
$$

for a.e $t \in(0, \infty)$. Here $\left|\mu^{\prime}(t)\right|$ is the metric derivative defined in 1.15 .

The general strategy of constructing curves of maximal slope is to use the JKO scheme, which we now describe.

Fix a time step $\tau>0$ and define $\mu_{\tau}^{0}=\mu_{0}$ where $\mu_{0}$ are the initial data. Then define iteratively

$$
\mu_{\tau}^{k+1} \in \operatorname{argmin}_{\mu \in \mathcal{P}_{2}(\mathcal{M})}\left[\frac{d_{W}^{2}\left(\mu, \mu_{\tau}^{k}\right)}{2 \tau}+\mathcal{E}(\mu)\right]
$$

We denote the piecewise constant interpolation by $\mu_{\tau}$. To be more precise, $\mu_{\tau}(0)=\mu_{0}$ and

$$
\mu_{\tau}(t)=\mu_{\tau}^{k+1}
$$

if $k \tau<t \leq(k+1) \tau$ for $k \geq 0$. The strategy is to show that there exists a subsequence $\tau_{n} \rightarrow 0$, such that $\tilde{\mu}^{n}=\mu_{\tau_{n}}$ converges narrowly to a curve of maximal slope $\mu$. Here in order to show the well-posedness of discrete scheme 4.2 and the convergence of the piecewise-constant interpolation to a curve a maximal slope, we apply the general theory developed in 2 . We now state and check that the conditions for the general theory to apply hold for our energy functional $\mathcal{E}$.

- Lower semicontinuity. $\mathcal{E}$ is sequentially lower semicontinuous with respect to narrow convergence of probability measures on $d_{W}$ bounded sets

$$
\sup _{m, n} d_{W}\left(\mu_{m}, \mu_{n}\right)<\infty, \mu_{n} \text { converges narrowly to } \mu \Rightarrow \liminf _{n \rightarrow \infty} \mathcal{E}\left(\mu_{n}\right) \geq \mathcal{E}(\mu) \text {. }
$$

In Section 2 we already show that $\mathcal{E}$ is lower semicontinuous with respect to narrow convergence of probability measures with uniformly bounded second moments.

- Coercivity. There exists $\tau_{*}>0$ and $\mu_{*} \in \mathcal{P}_{2}(\mathcal{M})$ such that

$$
\inf _{\mu \in \mathcal{P}_{2}(\mathcal{M})}\left\{\mathcal{E}(\mu)+\frac{1}{2 \tau_{*}} d_{W}^{2}\left(\mu, \mu_{*}\right)\right\}>-\infty .
$$


To prove coercivity, let $T$ be as in 3.1 and consider $x_{0} \in \mathcal{M}$ arbitrary. Then

$$
\begin{aligned}
& \mathcal{E}(\mu)+\frac{1}{2 \tau} d_{W}^{2}\left(\mu, \delta_{x_{0}}\right) \\
& =\int_{\mathcal{M}} V(x) d \mu(x)+\frac{1}{2} \int_{\mathcal{M} \times \mathcal{M}} W(x-y) d \mu(x) d \mu(y)+\frac{1}{2 \tau} \int_{\mathcal{M}} \operatorname{dist}^{2}\left(x, x_{0}\right) d \mu(x) \\
& \geq \int_{\mathcal{M}}\left(V\left(x_{0}\right)+\left\langle\nabla V, T\left(x_{0}, x\right)\right\rangle+\frac{\lambda}{2} \operatorname{dist}^{2}\left(x, x_{0}\right)\right) d \mu(x) \\
& +\int_{\mathcal{M} \times \mathcal{M}} \frac{\lambda}{2} \operatorname{dist}^{2}\left((x, y),\left(x_{0}, x_{0}\right)\right) d \mu(x) d \mu(y)+\frac{1}{2 \tau} \int_{\mathcal{M}} \operatorname{dist}^{2}\left(x, x_{0}\right) d \mu(x) \\
& =\int_{\mathcal{M}}\left(\left(\frac{3 \lambda}{2}+\frac{1}{2 \tau}\right) \operatorname{dist}^{2}\left(x_{0}, x\right)+\left\langle\nabla V\left(x_{0}\right), T\left(x_{0}, x\right)\right\rangle+V\left(x_{0}\right)\right) d \mu(x) .
\end{aligned}
$$

Notice that $\left\langle\nabla V\left(x_{0}\right), T\left(x_{0}, x\right)\right\rangle=g_{x_{0}}\left(A\left(x_{0}\right) \nabla V\left(x_{0}\right), T\left(x_{0}, x\right)\right)$ and

$$
g_{x_{0}}\left(T\left(x_{0}, x\right), T\left(x_{0}, x\right)\right)=\operatorname{dist}^{2}\left(x_{0}, x\right),
$$

so for any $\tau>0$ such that $\frac{3 \lambda}{2}+\frac{1}{2 \tau}>0$, i.e. for $3 \lambda^{-} \tau<1$, we have

$$
\inf _{\mu \in \mathcal{P}_{2}(\mathcal{M})}\left\{\mathcal{E}(\mu)+\frac{1}{2 \tau} d_{W}^{2}\left(\mu, \delta_{x_{0}}\right)\right\}>-\infty,
$$

which implies coercivity for $\mathcal{E}$.

- Compactness. Every $d_{W}$ bounded set contained in a sublevel of $\mathcal{E}$ is relatively compact with respect to the narrow convergence of probability measures

$$
\text { for }\left(\mu_{n}\right) \subset \mathcal{P}_{2}(\mathcal{M}) \text { with } \sup _{n} \mathcal{E}\left(\mu_{n}\right)<\infty \text { and } \sup _{m, n} d_{W}\left(\mu_{m}, \mu_{n}\right)<\infty \text {, }
$$

there exists a narrowly convergent subsequence of $\left(\mu_{n}\right)$.

To check Compactness condition, note that by Prokhorov's theorem, any sequence $\left(\mu_{n}\right) \subset$ $\mathcal{P}_{2}(\mathcal{M})$ such that $\sup _{m, n} d_{W}\left(\mu_{m}, \mu_{n}\right)<\infty, \mu_{n}$ has a narrowly convergent subsequence.

Thus we can apply Corollary 2.2.2 from [2] to show the existence of minimizers of 4.2 .

Lemma 4.2 (Existence of the discrete solutions). Suppose (M,g) satisfies assumptions (M1)-(M2) and $W, V$ satisfy (NL1)-(NL7). Then there exists $\tau_{0}>0$ depending only on $V, W$ such that for all $0<\tau<\tau_{0}$ and given $\nu \in \mathcal{P}_{2}(\mathcal{M})$, there exists $\mu_{\infty} \in \mathcal{P}_{2}(\mathcal{M})$ such that

$$
\mathcal{E}\left(\mu_{\infty}\right)+\frac{1}{2 \tau} d_{W}^{2}\left(\nu, \mu_{\infty}\right)=\inf _{\mu \in \mathcal{P}_{2}(\mathcal{M})}\left\{\mathcal{E}(\mu)+\frac{1}{2 \tau} d_{W}^{2}(\nu, \mu)\right\} .
$$

Proposition 2.2.3 from [2] provides the convergence of the scheme.

Proposition 4.3 (Compactness). There exist a limit curve $\mu \in A C_{l o c}^{2}\left([0, \infty) ; \mathcal{P}_{2}(\mathcal{M})\right)$ and a sequence $\tau_{n} \rightarrow 0^{+}$such that the piecewise constant interpolate $\tilde{\mu}^{n}=\mu_{\tau_{n}}$ defined as in (4.3) satisfies that $\tilde{\mu}^{n}(t)$ converges narrowly to $\mu(t)$ for any $t \in[0, \infty)$.

Note that by Lemma 3.2.2 from [2], we actually have a uniform bound on the second moments of $\tilde{\mu}^{n}$ :

$$
\sup _{n, \tau} \int_{\mathcal{M}} \operatorname{dist}^{2}\left(x, x_{0}\right) d \mu_{n}^{\tau}(x)<\infty .
$$

By the general theory developed in [2, the limit curve $\mu(t)$ is a curve of maximal slope with respect to upper gradient $\left|\partial^{-} \mathcal{E}\right|$, defined as

$$
\left|\partial^{-} \mathcal{E}\right|(\mu)=\inf \left\{\liminf _{n \rightarrow \infty}|\partial \mathcal{E}|\left(\mu_{n}\right): \mu_{n} \rightarrow \mu, \sup _{n}\left\{d_{W}\left(\mu_{n}, \mu\right), \mathcal{E}\left(\mu_{n}\right)\right\}<\infty\right\},
$$


where $\mu_{n} \rightarrow \mu$ means that $\mu_{n}$ converges narrowly to $\mu$. We still need to prove the lower semicontinuity of the slope to show that $\mu(\cdot)$ is a curve of maximal slope with respect to $|\partial \mathcal{E}|$ instead of $\left|\partial^{-} \mathcal{E}\right|$. We denote by $\kappa^{n}(t)$ the minimal subdifferential of $\mathcal{E}$ at $\tilde{\mu}^{n}(t)$. Section 3 gives

$$
\kappa^{n}(t, x)=-P_{x}\left(-A(x)\left(\int_{\mathcal{M}} \nabla W(x-y) d \tilde{\mu}^{n}(t, y)+\nabla V(x)\right)\right) .
$$

Theorem 4.4 (Lower semicontinuity of the slope). Assume that (M1)-(M2) and (NL1)-(NL7) hold true, then the metric slope of the piecewise constant interpolate $\tilde{\mu}^{n}$ satisfies that for a.e. $t>0$,

$$
\liminf _{n \rightarrow \infty}|\partial \mathcal{E}|^{2}\left(\tilde{\mu}^{n}(t)\right) \geq|\partial \mathcal{E}|^{2}(\mu(t)) .
$$

Remark 4.5. By Fatou's lemma, for any $T>0$

$$
\begin{aligned}
\liminf _{n \rightarrow \infty} \int_{0}^{T}|\partial \mathcal{E}|^{2}\left(\tilde{\mu}^{n}(t)\right) d t & \geq \int_{0}^{T} \liminf _{n \rightarrow \infty}|\partial \mathcal{E}|^{2}\left(\tilde{\mu}^{n}(t)\right) d t \\
& \geq \int_{0}^{T}|\partial \mathcal{E}|^{2}(\mu(t)) d t
\end{aligned}
$$

In the case $\partial \mathcal{M}=\emptyset$ and $W \in C^{1}\left(\mathbb{R}^{d} \backslash\{0\}\right)$, the lower semicontinuity of local slope can be proved as in Lemma 2.7 from [13. In the proof bellow, we allow that $\partial \mathcal{M} \neq \emptyset$.

In the case $\partial \mathcal{M} \neq \emptyset$, the argument in [13, does not work because the projection $P$ breaks the continuity and thus $\kappa^{n} d \mu^{n}$ does not necessarily converge narrowly to $\kappa d \mu$. However, the following useful observation holds:

Proposition 4.6. The function $\mathcal{M} \times \mathbb{R}^{d} \ni(x, \xi) \mapsto g_{x}\left(P_{x} \xi, P_{x} \xi\right)$ is lower semicontinuous. For all $x \in \mathcal{M}$, the function $\mathbb{R}^{d} \ni \xi \mapsto g_{x}\left(P_{x} \xi, P_{x} \xi\right)$ is convex.

Proof of Proposition. We first prove the lower semincontinuity property. Assume $\lim _{k \rightarrow \infty} x^{k}=x$ and $\lim _{k \rightarrow \infty} \xi^{k}=\xi$. If $\left\{x^{k}\right\}_{k=1}^{\infty} \subset \mathcal{M}$ then

$$
\begin{aligned}
g_{x}(P \xi, P \xi) & \leq g_{x}(\xi, \xi) \\
& =\lim _{k \rightarrow \infty} g_{x^{k}}\left(\xi^{k}, \xi^{k}\right) \\
& =\lim _{k \rightarrow \infty} g_{x^{k}}\left(P \xi^{k}, P \xi^{k}\right) .
\end{aligned}
$$

So lower semicontinuity is verified for $x \in \mathcal{M}$, since for such $x$ and any $\lim _{k \rightarrow \infty} x^{k}=x, x^{k} \in \mathcal{M}$ for all $k$ large enough. For $x \in \partial \mathcal{M}$, due to the fact above, it is enough to consider the case that $x^{k} \in \partial \mathcal{M}$ for all $k$. Let $\left\{e_{1}, \ldots, e_{d}\right\}$ be a continuous orthonormal basis of $T \mathcal{M}$ near $x$, such that on $\partial \mathcal{M}, e_{d}=\vec{n}$ where $\vec{n}$ is the unit outer normal vector with respect to the inner product $g$. We expand $\xi^{k}$ in this basis: $\xi^{k}=\sum_{i}^{d} \xi_{i}^{k} e_{i}\left(x^{k}\right)$. Then $P \xi^{k}=\sum_{i=1}^{d-1} \xi_{i}^{k} e_{i}\left(x^{k}\right)+\left(\xi_{d}^{k}\right)^{-} e_{d}\left(x^{k}\right)$ for $x^{k} \in \partial \mathcal{M}$. By the continuity of $g$ and $\mathcal{M}$, we have $\lim _{k \rightarrow \infty} \xi_{i}^{k}=\xi_{i}$ for all $1 \leq i \leq d$, thus

$$
\begin{aligned}
\lim _{k \rightarrow \infty} g_{x^{k}}\left(P \xi^{k}, P \xi^{k}\right) & =\lim _{k \rightarrow \infty}\left[\sum_{i=1}^{d-1}\left(\xi_{i}^{k}\right)^{2}+\left(\left(\xi_{d}^{k}\right)^{-}\right)^{2}\right] \\
& =\sum_{i=1}^{d-1} \xi_{i}^{2}+\left(\xi_{d}^{-}\right)^{2} \\
& =g_{x}(P \xi, P \xi) .
\end{aligned}
$$

We now turn to the convexity property. Similarly for $x \in \mathcal{M}$, since $P_{x} \xi=\xi$ for all $\xi \in \mathbb{R}^{d}$, it is straightforward to check that $\xi \mapsto g_{x}(\xi, \xi)$ is convex. So we assume $x \in \partial \mathcal{M}$. For any $\xi^{1}, \xi^{2} \in \mathbb{R}^{d}$, and $0 \leq \theta \leq 1$ we need to show that

$$
g_{x}\left(P_{x}\left((1-\theta) \xi^{1}+\theta \xi^{2}\right)\right) \leq(1-\theta) g_{x}\left(P_{x} \xi^{1}, P_{x} \xi^{1}\right)+\theta g_{x}\left(P_{x} \xi^{2}, P_{x} \xi^{2}\right) .
$$


Note that we only need to check that for the last coordinate, that is we only need to prove that

$$
\left(\left((1-\theta) \xi_{d}^{1}+\theta \xi_{d}^{2}\right)^{-}\right)^{2} \leq(1-\theta)\left(\left(\xi_{d}^{1}\right)^{-}\right)^{2}+\theta\left(\left(\xi_{d}^{2}\right)^{-}\right)^{2},
$$

which is a direct consequence of the fact that $f(x)=\left(x^{-}\right)^{2}$ is a convex function. The proposition is proved.

We now start to prove the lower semicontinuity of local slope

Proof of Theorem. Since $\kappa$ is the minimal subdifferential, be Remark 3.2 and Theorem 3.3 , we only need to prove

$$
\liminf _{n \rightarrow \infty} \int_{\mathcal{M}} g\left(\kappa^{n}(t, x), \kappa^{n}(t, x)\right) d \tilde{\mu}^{n}(t, x) \geq \int_{\mathcal{M}} g(\kappa(t, x), \kappa(t, x)) d \mu(t, x) .
$$

Note that the non-negative function $\mathcal{M} \times \mathbb{R}^{d} \ni(x, \xi) \mapsto g_{x}\left(P_{x} \xi, P_{x} \xi\right)$ satisfies the lower semicontinuity and convexity property. By Proposition 6.42 from [25], we know that for all $(x, \xi) \in \mathcal{M} \times \mathbb{R}^{d}$,

$$
g_{x}\left(P_{x} \xi, P_{x} \xi\right)=\sup _{i \in \mathbb{N}}\left\{a_{i}(x)+b_{i}(x) \xi\right\}
$$

for some bounded continuous functions $a_{i}, b_{i}$. A similar argument to one in Lemma 2.7 of 13 gives that $\nabla W * \mu^{n}$ converges narrowly to $\nabla W * \mu$. Thus we have for any $i \in \mathbb{N}$,

$$
\begin{aligned}
& \liminf _{n \rightarrow \infty} \int_{\mathcal{M}} g_{x}\left(\kappa^{n}(t, x), \kappa^{n}(t, x)\right) d \tilde{\mu}^{n}(t, x) \\
& =\liminf _{n \rightarrow \infty} \int_{\mathcal{M}} g_{x}\left(P\left(-\nabla W * \tilde{\mu}^{n}(t, x)-\nabla V(x)\right), P\left(-\nabla W * \tilde{\mu}^{n}(t, x)-\nabla V(x)\right)\right) d \tilde{\mu}^{n}(t, x) \\
& \geq \liminf _{n \rightarrow \infty} \int_{\mathcal{M}}\left(a_{i}(x)-b_{i}(x)\left(\nabla W * \tilde{\mu}^{n}(t)(x)+\nabla V(x)\right)\right) d \tilde{\mu}^{n}(t, x) \\
& =\int_{\mathcal{M}}\left(a_{i}(x)-b_{i}(x)(\nabla W * \mu(t)(x)+\nabla V(x))\right) d \mu(t, x) .
\end{aligned}
$$

Taking supremum over $i \in \mathbb{N}$ and using Lebesgue's monotone convergence theorem then gives

$$
\begin{aligned}
\liminf _{n \rightarrow \infty} \int_{\mathcal{M}} g\left(\kappa^{n}(t, x), \kappa^{n}(t, x)\right) d \tilde{\mu}^{n}(t, x) & \geq \sup _{i \in \mathbb{N}} \int_{\mathcal{M}}\left(a_{i}(x)-b_{i}(x)(\nabla W * \mu(t)(x)+\nabla V(x))\right) d \mu(t, x) \\
& =\int_{\mathcal{M}} g(\kappa(t, x), \kappa(t, x)) d \mu(t, x) .
\end{aligned}
$$

We can now give the main result of this section.

Theorem 4.7 (Existence of curves of maximal slope). Suppose $(\mathcal{M}, g)$ satisfies (M1)-(M2) and $W, V$ satisfy (NL1)-(NL7). Then there exists at least one curve of maximal slope for the functional $\mathcal{E}$, i.e., there exists $\mu \in A C_{\text {loc }}\left([0, \infty) ; \mathcal{P}_{2}(\mathcal{M})\right)$ such that for all $T \geq 0$

$$
\mathcal{E}\left(\mu_{0}\right) \geq \mathcal{E}(\mu(T))+\frac{1}{2} \int_{0}^{T}\left|\mu^{\prime}\right|^{2}(t) d t+\frac{1}{2} \int_{0}^{T}|\partial \mathcal{E}|^{2}(\mu(t)) d t .
$$

Proof. We know that $\mu \mapsto \mathcal{E}(\mu)$ and $\mu \mapsto \int_{0}^{T}|\partial \mathcal{E}|^{2}(\mu(t)) d t$ are lower semicontinuous with respect to the narrow convergence. Thus we only need to prove

$$
\liminf _{n \rightarrow \infty} \int_{0}^{T}\left|\left(\tilde{\mu}^{n}\right)^{\prime}\right|^{2}(t) d t \geq \int_{0}^{T}\left|\mu^{\prime}\right|^{2}(t) d t
$$

which comes from estimates of JKO scheme in general metric space, see for example, Corollary 3.3.4 from [2]. 


\section{EXISTENCE OF THE GRADIENT FLOW}

In this section, we first show that locally absolutely continuous curves in $\mathcal{P}_{2}(\mathcal{M})$ with respect to $d_{W}$ are solutions to continuity equations in the sense of distributions. Furthermore velocities are in $L^{2}(g, \mu)$ and belonging to the tangent space to the set of configurations. We then prove the existence of gradient flow and the stability property of the gradient flow.

Lemma 5.1. Let $\mu(t)$ be an absolutely continuous curve in $\mathcal{P}_{2}(\mathcal{M})$ and $\gamma_{t}^{h} \in \Gamma_{o}(\mu(t), \mu(t+h))$ be an optimal plan between $\mu(t)$ and $\mu(t+h)$. Denote the disintegration of $\gamma_{t}^{h}$ with respect to $\mu(t)$ by $\nu_{x}^{h}$, then $\int_{\mathcal{M}} \frac{T(x, y)}{h} d \nu_{x}^{h}(y)$ converges weakly in $L^{2}(g, \mu(t))$ to a vector field $v(t, x)$ for a.e. $t>0$ such that $\mu(t)$ satisfy the continuity equation

$$
\frac{\partial}{\partial t} \mu(t, x)+\operatorname{div}(\mu(t, x) v(t, x))=0
$$

in the sense of distributions, i.e., test against $\phi \in C_{c}^{\infty}([0, \infty) \times \mathcal{M})$, and

$$
\int_{\mathcal{M}} g(v(t, x), v(t, x)) d \mu(t, x)=\left|\mu^{\prime}\right|^{2}(t)
$$

for a.e. $t>0$.

Proof. For the existence of a unique minimal $L^{2}(g, \mu(t))$-norm vector field $v(t)$ such that $\mu(t)$ satisfies (5.1) and (5.2), we refer to Theorem 2.29 from [1]. We now show that such $v$ is given by the limit of $\int_{\mathcal{M}} \frac{T(x, y)}{h} d \nu_{x}^{h}(y)$. Note that

$$
\begin{aligned}
\int_{\mathcal{M}} g_{x}\left(\int_{\mathcal{M}} \frac{T(x, y)}{h} d \nu_{x}^{h}(y), \int_{\mathcal{M}} \frac{T(x, y)}{h} d \nu_{x}^{h}(y)\right) d \mu(t, x) & \leq \int_{\mathcal{M} \times \mathcal{M}} g_{x}\left(\frac{T(x, y)}{h}, \frac{T(x, y)}{h}\right) d \nu_{x}^{h}(y) d \mu(t, x) \\
& =\int_{\mathcal{M} \times \mathcal{M}} g_{x}\left(\frac{T(x, y)}{h}, \frac{T(x, y)}{h}\right) d \gamma_{t}^{h}(x, y) \\
& =\frac{1}{h^{2}} d_{W}^{2}(\mu(t), \mu(t+h)) .
\end{aligned}
$$

Since $\mu(t)$ is absolutely continuous, we know that $\frac{1}{h^{2}} d_{W}^{2}(\mu(t), \mu(t+h)) \leq C$ uniformly in $h$ for some constant $C$. Thus, uniformly in $h$, we have

$$
\int_{\mathcal{M}} g_{x}\left(\int_{\mathcal{M}} \frac{T(x, y)}{h} d \nu_{x}^{h}(y), \int_{\mathcal{M}} \frac{T(x, y)}{h} d \nu_{x}^{h}(y)\right) d \mu(t, x) \leq C .
$$

So there exist a vector field $\tilde{v}(t, x)$ and a sequence $\left\{h_{n}\right\}$ converging to 0 , such that $\int_{\mathcal{M}} \frac{T(x, y)}{h_{n}} d \nu_{x}^{h_{n}}(y)$ converges weakly in $L^{2}(g, \mu(t, x))$ to $\tilde{v}$. We claim that

$$
\lim _{n \rightarrow \infty} \frac{\int_{\mathcal{M}} \phi(t, x) d \mu\left(t+h_{n}, x\right)-\int_{\mathcal{M}} \phi(t, x) d \mu(t, x)}{h_{n}}=\int_{\mathcal{M}} g_{x}(\nabla \phi(t, x), \tilde{v}(t, x)) d \mu(t, x),
$$

for a.e. $t>0$ and for any $\phi \in C_{c}^{\infty}\left([0, \infty) \times \mathbb{R}^{n}\right)$.

Indeed, for the left-hand side we know,

$$
\begin{aligned}
\frac{\int_{\mathcal{M}} \phi(t, x) d \mu\left(t+h_{n}, x\right)-\int_{\mathcal{M}} \phi(t, x) d \mu(t, x)}{h_{n}} & =\frac{1}{h_{n}} \int_{\mathcal{M} \times \mathcal{M}}(\phi(t, y)-\phi(t, x)) d \gamma_{t}^{h_{n}}(x, y) \\
& =\frac{1}{h_{n}} \int_{\mathcal{M} \times \mathcal{M}}\langle\nabla \phi(t, x), T(x, y)\rangle d \gamma_{t}^{h_{n}}(x, y)+o\left(h_{n}\right) \\
& =\int_{\mathcal{M}}\left\langle\nabla \phi(t, x), \int_{\mathcal{M}} \frac{T(x, y)}{h_{n}} d \nu_{x}^{h_{n}}(y)\right\rangle d \mu(t, x)+o\left(h_{n}\right) \\
& =\int_{\mathcal{M}} g_{x}\left(A(x) \nabla \phi(t, x), \int_{\mathcal{M}} \frac{T(x, y)}{h_{n}} d \nu_{x}^{h_{n}}(y)\right) d \mu(t, x)+o\left(h_{n}\right)
\end{aligned}
$$


Since $\int_{\mathcal{M}} \frac{T(x, y)}{h_{n}} d \nu_{x}^{h_{n}}(y)$ converges weakly in $L^{2}(g, \mu(t))$ to $\tilde{v}(t, x)$ and $A(x) \nabla \phi(t, x) \in L^{2}(g, \mu(t))$, we get

$$
\begin{aligned}
\lim _{n \rightarrow \infty} \frac{\int_{\mathcal{M}} \phi(t, x) d \mu\left(t+h_{n}, x\right)-\int_{\mathcal{M}} \phi(t, x) d \mu(t, x)}{h_{n}} & =\int_{\mathcal{M}} g_{x}(A(x) \nabla \phi(t, x), \tilde{v}(t, x)) d \mu(t, x) \\
& =\int_{\mathcal{M}}\langle\nabla \phi(t, x), \tilde{v}(t, x)\rangle d \mu(t, x) .
\end{aligned}
$$

Since $\mu(t)$ satisfies 5.1 with respect to the vector field $v$, we know that

$$
\lim _{h \rightarrow 0} \frac{\int_{\mathcal{M}} \phi(t, x) d \mu(t+h, x)-\int_{\mathcal{M}} \phi(t, x) d \mu(t, x)}{h}=\int_{\mathcal{M}} g_{x}(\nabla \phi(t, x), v(t, x)) d \mu(t, x),
$$

for a.e. $t>0$. Thus $\int_{\mathcal{M}} g_{x}(\nabla \phi(t, x), v(t, x)-\tilde{v}(t, x)) d \mu(t, x)=0$ for a.e. $t>0$ and $\mu(t)$ satisfies (5.1) with respect to $\tilde{v}(t)$. Now notice that

$$
\begin{aligned}
\int_{\mathcal{M}} g_{x}(\tilde{v}(t, x), \tilde{v}(t, x)) d \mu(t, x) & \leq \lim _{n \rightarrow \infty} \int_{\mathcal{M}} g_{x}\left(\int_{\mathcal{M}} \frac{T(x, y)}{h_{n}} d \nu_{x}^{h_{n}}(y), \int_{\mathcal{M}} \frac{T(x, y)}{h_{n}} d \nu_{x}^{h_{n}}(y)\right) d \mu(t, x) \\
& \leq \lim _{n \rightarrow \infty} \frac{1}{h_{n}^{2}} d_{W}^{2}\left(\mu(t), \mu\left(t+h_{n}\right)\right) \\
& =\left|\mu^{\prime}\right|^{2}(t)=\int_{\mathcal{M}} g_{x}(v(t, x), v(t, x)) d \mu(t, x) .
\end{aligned}
$$

Together with the minimal $L^{2}(g, \mu(t))$-norm property of $v$, we have $\tilde{v}(t)=v(t)$. Since for any $h_{n} \rightarrow 0$ such that $\lim _{n \rightarrow \infty} \int_{\mathcal{M}} \frac{T(x, y)}{h_{n}} d \nu_{x}^{h_{n}}(y)$ converges weakly in $L^{2}(g, \mu(t))$, the weak limit is the same $v(t)$, we have $\int_{\mathcal{M}} \frac{T(x, y)}{h} d \nu_{x}^{h}(y)$ converges weakly in $L^{2}(g, \mu(t))$ to $v(t, x)$. The lemma is proved.

We will call $v(t)$ the tangent velocity field of $\mu(t)$, now we can define gradient flow by

Definition 5.2 (Gradient flows). A locally absolutely continuous curve $[0, \infty) \ni t \mapsto \mu(t) \in \mathcal{P}_{2}(\mathcal{M})$ is a gradient flow with respect to $\mathcal{E}$ if for a.e. $t>0$

$$
v(t) \in-\partial \mathcal{E}(\mu(t)),
$$

where $v(t)$ is the tangent velocity field for $\mu(t)$.

Then we can show the proof of Theorem 1.3

Proof of Theorem 1.3. We only need to prove the following chain rule

$$
\frac{d}{d t} \mathcal{E}(\mu(t))=\int_{\mathcal{M}} g_{x}(\kappa(t, x), v(t, x)) d \mu(t, x),
$$

for a.e. $t>0$, where $v(t)$ is the tangent velocity field for the absolutely continuous curve $\mu(t)$. Indeed, the fact that $\mu(t)$ is a curve of maximal slope implies

$$
\frac{d}{d t} \mathcal{E}(\mu(t)) \leq-\frac{1}{2} \int_{\mathcal{M}} g_{x}(v(t, x), v(t, x)) d \mu(t, x)-\frac{1}{2} \int_{\mathcal{M}} g_{x}(\kappa(t, x), \kappa(t, x)) d \mu(t, x) .
$$

If (5.4) holds, then together with (5.5), we have $v(t, x)=-\kappa(t, x)$ for a.e. $t>0$ and $\mu(t)$ is a gradient flow with respect to $\mathcal{E}$. Then by Lemma 5.1, $\mu(t)$ is a weak measure solution to 1.9 with initial data $\mu_{0}$.

We now prove the chain rule 5.4 . Since $\kappa(t) \in \partial \mathcal{E}(\mu(t))$, we know

$$
\mathcal{E}(\mu(t+h)) \geq \mathcal{E}(\mu(t))+\int_{M \times M} g(\kappa(t, x), T(x, y)) d \gamma_{t}^{h}(x, y)+o\left(d_{W}(\mu(t), \mu(t+h))\right) .
$$


For $h>0$, we have

$$
\begin{aligned}
\lim _{h \rightarrow 0^{+}} \frac{\mathcal{E}(\mu(t+h))-\mathcal{E}(\mu(t))}{h} & \geq \int_{\mathcal{M} \times \mathcal{M}} g_{x}\left(\kappa(t, x), \frac{T(x, y)}{h}\right) d \gamma_{t}^{h}(x, y) \\
& =\int_{\mathcal{M}} g_{x}\left(\kappa(t, x), \int_{\mathcal{M}} \frac{T(x, y)}{h} d \nu_{x}^{h}(y)\right) d \mu(t, x) \\
& =\int_{\mathcal{M}} g_{x}(\kappa(t, x), v(t, x)) d \mu(t, x) .
\end{aligned}
$$

Similarly, for $h<0$, we have

$$
\lim _{h \rightarrow 0^{-}} \frac{\mathcal{E}(\mu(t+h))-\mathcal{E}(\mu(t))}{h} \leq \int_{\mathcal{M}} g_{x}(\kappa(t, x), v(t, x)) d \mu(t, x) .
$$

Note that the function $t \rightarrow \mathcal{E}(\mu(t))$ is non-increasing, thus differentiable for a.e. $t>0$, so

$$
\frac{d}{d t} \mathcal{E}(\mu(t))=\int_{\mathcal{M}} g_{x}(\kappa(t, x), v(t, x)) d \mu(t, x),
$$

for a.e. $t>0$ as desired. Also since $\partial^{o} \mathcal{E}(\mu(t))=v(t, x)=-\kappa(t, x)$, 1.17) is true. To prove (1.18), we only need to show that $\mathcal{E}(\mu(t))$ is locally absolutely continuous. We note that by the linear growth conditions on $\nabla V$ (1.11) and $\nabla W(1.10),|V(x)-V(y)| \leq C(1+\operatorname{dist}(x, y)) \operatorname{dist}(x, y)$ and $|W(x-z)-W(y-w)| \leq C(1+\operatorname{dist}(x, y)+\operatorname{dist}(z, w))(\operatorname{dist}(x, y)+\operatorname{dist}(z, w))$. Then for $0 \leq s<t<\infty$ and $\gamma \in \Gamma_{o}(\mu(t), \mu(s))$ an optimal plan,

$$
\begin{aligned}
|\mathcal{E}(\mu(t))-\mathcal{E}(\mu(s))| & \leq \int_{\mathcal{M} \times \mathcal{M}} C(1+\operatorname{dist}(x, y)) \operatorname{dist}(x, y) d \gamma(x, y) \\
& \leq C\left(1+d_{W}(\mu(t), \mu(s)) d_{W}(\mu(t), \mu(s)) .\right.
\end{aligned}
$$

Thus $\mathcal{E}(\mu(t))$ is locally absolutely continuous since $\mu(t)$ is locally absolutely continuous in $\left(\mathcal{P}_{2}(\mathcal{M}), d_{W}\right)$.

Next, we start to prove Theorem 1.4 that $\lambda$-convexity of $\mathcal{E}$ implies the stability of the gradient flow. Before proving the theorem, we need the following

Lemma 5.3. Let $\mu(t)$ be a locally absolutely continuous curve in $\mathcal{P}_{2}(\mathcal{M})$ with tangent velocity $v$, then for a.e. $t>0$,

$$
\frac{1}{2} \frac{d}{d t} d_{W}^{2}(\mu(t), \nu)=-\int_{\mathcal{M} \times \mathcal{M}} g_{x}(v(t, x), T(x, y)) d \gamma_{t}(x, y),
$$

for any fixed $\nu \in \mathcal{P}_{2}(\mathcal{M})$ and $\gamma_{t} \in \Gamma_{o}(\mu(t), \nu)$ an optimal plan.

Proof of Lemma. We first notice that the function $t \mapsto d_{W}^{2}(\mu(t), \nu)$ is differentiable for a.e. $t>0$ since $t \mapsto \mu(t)$ is locally absolutely continuous in $\left(\mathcal{P}_{2}(\mathcal{M}), d_{W}\right)$. In the rest of the proof, we assume that we are working on $t>0$ such that the function $s \mapsto \frac{1}{2} d_{W}^{2}(\mu(s), \nu)$ is differentiable at $t$. In the case $v$ is locally Lipschitz in space and $\mathcal{M}$ has no boundary then using the flow map with velocity field $v$, similar arguments as in [45, 19] imply (5.6). However, in our case, we need to deal with the fact that since $v$ is not continuous the flow map is not readily available and furthermore that a geodesic in direction $v$ may not exist at the boundary. We divide the proof into two steps.

Step 1. Consider the case that $\mu(t), \nu$ have compact support for all $t>0$. To show (5.6) we modify the arguments of Theorem 8.4.7 from [2. An issue is that, as in the proof of Theorem 3.3 there may exist $x \in \partial \mathcal{M}$ such that there exists no $t>0$ for which $\exp _{x}(t v(x)) \in \mathcal{M}$ exists. To deal with 
this problem we use the following approximations. For $h \in \mathbb{R}$ with $|h|$ small, define

$$
v_{h}(t, x)= \begin{cases}v(t, x), & \text { if } x \in B\left(\frac{1}{|h|}\right) \cap \mathcal{M}_{|h|} \\ v(t, x)-h n(x), & \text { if } x \in B\left(\frac{1}{|h|}\right) \cap \partial \mathcal{M} \\ 0, & \text { otherwise. }\end{cases}
$$

It is direct to check that $v_{h}$ converges to $v$ in $L^{2}(g, \mu(t))$. For fixed $h \in \mathbb{R}$, same argument as in the proof of Theorem 3.3 shows that there exists $C(h)>0$ such that $\exp _{x}\left(t h v_{h}(t, x)\right)$ exists for all $0 \leq t \leq C(h)$ and $x \in \mathcal{M}$. Thus there exists a function $f$ such that $\lim _{h \rightarrow 0} f(h)=0$ and $\exp _{x}\left(h v_{f(h)}(t, x)\right) \in \mathcal{M}$ for all $x \in \mathcal{M}$. We claim that for a.e. $t>0$

$$
\lim _{h \rightarrow 0} \frac{d_{W}^{2}\left(\left(\exp \left(h v_{f(h)}\right)\right)_{\sharp} \mu(t), \mu(t+h)\right)}{h^{2}}=0 .
$$

Indeed, if the claim is true, then for a.e. $t>0$, we know that $d_{W}^{2}(\mu(t), \nu)$ is differentiable and

$$
\begin{aligned}
\frac{d}{d t} d_{W}^{2}(\mu(t), \nu) & =\lim _{h \rightarrow 0} \frac{d_{W}^{2}(\mu(t+h), \nu)-d_{W}^{2}(\mu(t), \nu)}{h} \\
& =\lim _{h \rightarrow 0} \frac{d_{W}^{2}\left(\left(\exp \left(h v_{f(h)}\right)\right)_{\sharp} \mu(t), \nu\right)-d_{W}^{2}(\mu(t), \nu)}{h} .
\end{aligned}
$$

Since $\left(\exp \left(h v_{f(h)}\right), i d\right)_{\sharp} \gamma_{t} \in \Gamma\left(\exp \left(h v_{f(h)}\right)_{\sharp} \mu(t), \nu\right)$, we get

$$
d_{W}^{2}\left(\left(\exp \left(h v_{f(h)}\right)\right)_{\sharp} \mu(t), \nu\right) \leq \int_{\mathcal{M} \times \mathcal{M}} \operatorname{dist}^{2}\left(\exp _{x}\left(h v_{f(h)}(t, x)\right), y\right) d \gamma_{t}(x, y) .
$$

Recall that by the first variation formula, for any $x, y \in \mathcal{M}$, denote

$$
D(x, y)=\left\{v \in T_{x} \mathcal{M}: \exp _{x}(t v) \in \mathcal{M} \forall t \in[0,1], \exp _{x}(v)=y, g_{x}(v, v)=\operatorname{dist}^{2}(x, y)\right\},
$$

then

$$
\lim _{h \rightarrow 0^{+}} \frac{\operatorname{dist}^{2}\left(\exp _{x}(h \xi), y\right)-\operatorname{dist}^{2}(x, y)}{h}=\min \left\{-2 g_{x}(\xi, v): v \in D(x, y)\right\} .
$$

So taking $h \rightarrow 0^{+}$and using the Lebesgue's dominated convergence theorem yields

$$
\begin{aligned}
\frac{d^{+}}{d t} d_{W}^{2}(\mu(t), \nu) & \leq \lim _{h \rightarrow 0^{+}} \frac{1}{h} \int_{\mathcal{M} \times \mathcal{M}}\left(\operatorname{dist}^{2}\left(\exp _{x}\left(h v_{f(h)}(t, x)\right), y\right)-\operatorname{dist}^{2}(x, y)\right) d \gamma_{t}(x, y) \\
& \leq-2 \int_{\mathcal{M} \times \mathcal{M}} g_{x}(v(t, x), T(x, y)) d \gamma_{t}(x, y) .
\end{aligned}
$$

Similarly, taking $h \rightarrow 0^{-}$gives

$$
\frac{d^{-}}{d t} d_{W}^{2}(\mu(t), \nu) \geq-2 \int_{\mathcal{M} \times \mathcal{M}} g_{x}(v(t, x), T(x, y)) d \gamma_{t}(x, y) .
$$

Thus we have

$$
\frac{1}{2} \frac{d}{d t} d_{W}^{2}(\mu(t), \nu)=-\int_{\mathcal{M} \times \mathcal{M}} g_{x}(v(t, x), T(x, y)) d \gamma_{t}(x, y),
$$

for a.e. $t>0$.

We now prove the claim. It is enough to show that

$$
\lim _{h \rightarrow 0} \int_{\mathcal{M} \times \mathcal{M}} \frac{1}{h^{2}} \operatorname{dist}^{2}\left(\exp _{x}\left(h v_{f(h)}(t, x)\right), y\right) d \gamma_{t}^{h}(x, y)=0
$$


where $\gamma_{t}^{h} \in \Gamma_{o}(\mu(t), \mu(t+h))$. Since $\mu(t)$ has compact support for all $t>0$, we only need to show 5.9) for compact subsets of $\mathcal{M}$, i.e., to show

$$
\lim _{h \rightarrow 0} \int_{K \times K} \frac{1}{h^{2}} \operatorname{dist}^{2}\left(\exp _{x}\left(h v_{f(h)}(t, x)\right), y\right) d \gamma_{t}^{h}(x, y)=0
$$

for any compact subset $K \Subset \mathcal{M}$. On $K$, we have that the sectional curvature is bounded from below say by $-k$, then by rescaling, we may assume the constant for the lower bounded of sectional curvature is -1 . By comparison theorem, refer to 38 Theorem 79 , we have

$$
\begin{aligned}
& \cosh \left[\operatorname{dist}\left(\exp _{x}\left(h v_{f(h)}(t, x)\right), y\right)\right] \\
& \leq \cosh [\operatorname{dist}(x, y)] \cosh \left[h\left|v_{f(h)}(t, x)\right|_{g}\right]-\sinh [\operatorname{dist}(x, y)] \sinh \left[h\left|v_{f(h)}(t, x)\right|_{g}\right] \cos \alpha,
\end{aligned}
$$

where $\alpha$ is angle between $v_{f(h)}(t, x)$ and $T(x, y)$, i.e., $\cos \alpha=\frac{g_{x}\left(v_{f(h)}(t, x), T(x, y)\right)}{\operatorname{dist}(x, y)\left|v_{f(h)}(t, x)\right|_{g}}$. Note that

$$
\cosh [z]=1+\frac{1}{2} z^{2}+O\left(z^{4}\right)
$$

and

$$
\sinh [z]=z+O\left(z^{3}\right)
$$

Expanding cosh, sinh in the comparison formula, we have

$$
\begin{aligned}
& 1+\frac{1}{2} \operatorname{dist}^{2}\left(\exp _{x}\left(h v_{f(h)}(t, x)\right), y\right) \\
& \leq \cosh \left[\operatorname{dist}\left(\exp _{x}\left(h v_{f(h)}(t, x)\right), y\right)\right] \\
& \leq 1+\frac{1}{2} \operatorname{dist}^{2}(x, y)+\frac{1}{2} h^{2}\left|v_{f(h)}(t, x)\right|_{g}^{2}-h \operatorname{dist}(x, y)\left|v_{f(h)}(t, x)\right|_{g} \cos \alpha+O\left(h^{3}\right)+O\left(\operatorname{dist}^{3}(x, y)\right) .
\end{aligned}
$$

Thus

$$
\begin{aligned}
& \lim _{h \rightarrow 0} \int_{K \times K} \frac{1}{h^{2}} \operatorname{dist}^{2}\left(\exp _{x}\left(h v_{f(h)}(t, x)\right), y\right) d \gamma_{t}^{h}(x, y) \\
& \leq \lim _{h \rightarrow 0} \int_{K \times K}\left(\frac{1}{h^{2}} \operatorname{dist}^{2}(x, y)-2 \frac{1}{h} \operatorname{dist}(x, y)\left|v_{f(h)}(t, x)\right|_{g} \cos \alpha+\left|v_{f(h)}(t, x)\right|_{g}^{2}+o(h)\right) d \gamma_{t}^{h}(x, y) \\
& =\lim _{h \rightarrow 0} \int_{K \times K}\left(\frac{1}{h^{2}} \operatorname{dist}^{2}(x, y)-2 g_{x}\left(\frac{T(x, y)}{h}, v_{f(h)}(t, x)\right)+\left|v_{f(h)}(t, x)\right|_{g}^{2}\right) d \gamma_{t}^{h}(x, y) \\
& =\lim _{h \rightarrow 0} \int_{K \times K} g_{x}\left(\frac{T(x, y)}{h}-v_{f(h)}(t, x), \frac{T(x, y)}{h}-v_{f(h)}(t, x)\right) d \gamma_{t}^{h}(x, y) \\
& =0 .
\end{aligned}
$$

Step 2. 5.6 holds for general $\mu(t), \nu \in \mathcal{P}_{2}(\mathcal{M})$. To show that, we need to perform the same approximation as in the proof of Theorem 23.9 from [45, which requires that notion of dynamical coupling, refer to 45. Here we sketch the approximation and argument, let $A_{k}=\left\{\gamma: \sup _{t} \operatorname{dist}(z, \gamma(t)) \leq k\right\}$, where $\gamma$ is a random curve $\gamma:[0,1] \rightarrow \mathcal{M}$ and $e_{t}$ is the evaluation map $e_{t}(\gamma)=\gamma(t)$. Define $\mu^{k}(t)=\left(e_{t}\right)_{\sharp} \Pi_{k}$ where $\Pi_{k}(d \gamma)=\frac{\chi_{\gamma \in A_{k}} \Pi(d \gamma)}{\Pi\left(A_{k}\right)}$ and $\Pi$ is a probability measure on the action minimizing curves. Denote $Z_{k}=\Pi\left(A_{k}\right)$ then $Z_{k} \uparrow 1, Z_{k} \mu^{k}(t) \uparrow \mu(t)$ as $k \rightarrow \infty$. For each $k \mu^{k}$ solves

$$
\frac{\partial \mu^{k}(t)}{\partial t}+\operatorname{div}\left(\mu^{k}(t) v(t)\right)=0
$$

and $\mu^{n}(t)$ has compact support in $B(z, k)$. So by Step 1,

$$
\frac{1}{2} \frac{d}{d t} d_{W}^{2}\left(\mu^{k}(t), \nu^{k}\right)=-\int_{\mathcal{M} \times \mathcal{M}} g_{x}(v(t, x), T(x, y)) d \gamma_{t}^{k}(x, y) .
$$


Since $d_{W}^{2}\left(\mu^{k}(t), \nu^{k}\right)$ is locally absolutely continuous, integrating gives

$$
\frac{d_{W}^{2}\left(\mu^{k}(t), \nu^{k}\right)}{2}=\frac{d_{W}^{2}\left(\mu^{k}(0), \nu^{k}\right)}{2}-\int_{0}^{t} \int_{\mathcal{M} \times \mathcal{M}} g_{x}(v(s, x), T(x, y)) d \gamma_{s}^{k}(x, y) d s .
$$

The only thing left is to take $k \rightarrow \infty$. By the proof of Theorem 23.9 [45], $d_{W}\left(\mu^{k}(t), \mu(t)\right)=0$ and we only need to check

$$
\lim _{k \rightarrow \infty} \int_{0}^{t} \int_{\mathcal{M} \times \mathcal{M}} g_{x}(v(s, x), T(x, y)) d \gamma_{s}^{k}(x, y) d s=\int_{0}^{t} \int_{\mathcal{M} \times \mathcal{M}} g_{x}(v(s, x), T(x, y)) d \gamma_{s}(x, y) d s .
$$

Notice that

$$
\begin{aligned}
& \left|\int_{\mathcal{M} \times \mathcal{M}} g_{x}(v(s, x), T(x, y)) d \gamma_{s}^{k}(x, y)\right| \\
& \leq\left(\int_{\mathcal{M} \times \mathcal{M}} \operatorname{dist}^{2}(x, y) d \gamma_{s}^{k}(x, y)\right)^{\frac{1}{2}}\left(\int_{\mathcal{M} \times \mathcal{M}} g_{x}(v(s, x), v(s, x)) d \gamma_{s}^{k}(x, y)\right)^{\frac{1}{2}} \\
& \leq d_{W}\left(\mu^{k}(s), \nu^{k}\right)\left(\frac{1}{Z_{k}} \int_{\mathcal{M}} g_{x}(v(s, x), v(s, x)) d \mu^{k}(s, x)\right)^{\frac{1}{2}} \\
& \leq C\left(\int_{\mathcal{M}} g_{x}(v(s, x), v(s, x)) d \mu(s, x)\right)^{\frac{1}{2}},
\end{aligned}
$$

and $\int_{\mathcal{M}} g_{x}(v(s, x), v(s, x)) d \mu(s, x) \in L^{1}([0, t])$. It is then sufficient to prove that for a.e. $s \in(0, t)$

$$
\int_{\mathcal{M} \times \mathcal{M}} g_{x}(v(s, x), T(x, y)) d \gamma_{s}^{k}(x, y) \rightarrow \int_{\mathcal{M} \times \mathcal{M}} g_{x}(v(x, s), T(x, y)) d \gamma_{s}(x, y) .
$$

Since

$$
\begin{aligned}
& \int_{\mathcal{M} \times \mathcal{M}}\left|g_{x}(v(s, x), T(x, y))\right| d\left|\gamma_{s}^{k}-\gamma_{s}\right|(x, y) \\
& \leq\left(\int_{\mathcal{M} \times \mathcal{M}} g_{x}(v(s, x), v(s, x)) d\left|\gamma_{s}^{k}-\gamma_{s}\right|(x, y)\right)^{\frac{1}{2}}\left(\int_{\mathcal{M} \times \mathcal{M}} \operatorname{dist}^{2}(x, y) d\left|\gamma_{s}^{k}-\gamma_{s}\right|(x, y)\right)^{\frac{1}{2}} \\
& \leq C d_{W}(\mu(s), \nu)\left(\int_{\mathcal{M}} g_{x}(v(s, x), v(s, x)) d\left|\mu^{k}(s)-\mu(s)\right|(x)\right)^{\frac{1}{2}},
\end{aligned}
$$

and

$$
\begin{aligned}
& \int_{\mathcal{M}} g_{x}(v(s, x), v(s, x)) d\left|\mu^{k}(s)-\mu(s)\right|(x) \\
& \leq\left(Z_{k}^{-1}-1\right) \int_{\mathcal{M}} g_{x}(v(s, x), v(s, x)) d \mu(s, x)+Z_{k}^{-1} \int_{\mathcal{M}} g_{x}(v(s, x), v(s, x)) d\left|Z_{k} \mu^{k}(s)-\mu(s)\right|(x) \\
& \leq\left(Z_{k}^{-1}-1\right) \int_{\mathcal{M}} g_{x}(v(s, x), v(s, x)) d \mu(s, x)+Z_{k}^{-1} \int_{e_{s}(S) \backslash e_{s}\left(A_{k}\right)} g_{x}(v(s, x), v(s, x)) d \mu(s, x) \\
& \leq\left(Z_{k}^{-1}-1\right) \int_{\mathcal{M}} g_{x}(v(s, x), v(s, x)) d \mu(s, x)+Z_{k}^{-1} \int_{S \backslash A_{k}} g_{\gamma(s)}(v(s, \gamma(s)), v(s, \gamma(s)) d \Pi(\gamma),
\end{aligned}
$$

we know

$$
\lim _{k \rightarrow \infty} \int_{\mathcal{M} \times \mathcal{M}}\left|g_{x}(v(s, x), T(x, y))\right| d\left|\gamma_{s}^{k}-\gamma_{s}\right|(x, y)=0 .
$$

Thus 5.12 holds true. Take $k \rightarrow \infty$ in (5.11) then gives (5.6).

We now prove Theorem 1.4 
Proof of Theorem 1.4. Let $\kappa^{1} \in \partial^{o} \mathcal{E}\left(\mu^{1}(t)\right), \kappa^{2} \in \partial^{o} \mathcal{E}\left(\mu^{2}(t)\right)$ be the minimal subdifferentials and $v^{1}, v^{2}$ be the tangent velocities of the absolutely continuous curves $\mu^{1}(t), \mu^{2}(t)$ respectively. Also denote $\gamma_{t} \in \Gamma_{o}\left(\mu^{1}(t), \mu^{2}(t)\right)$ an optimal plan between $\mu^{1}(t)$ and $\mu^{2}(t)$. By the definition of subdifferential, we know that

$$
\mathcal{E}\left(\mu^{2}(t)\right) \geq \mathcal{E}\left(\mu^{1}(t)\right)+\int_{\mathcal{M} \times \mathcal{M}} g\left(\kappa^{1}(t, x), T(x, y)\right) d \gamma_{t}(x, y)+\frac{\lambda}{2} d_{W}^{2}\left(\mu^{1}(t), \mu^{2}(t)\right),
$$

and

$$
\mathcal{E}\left(\mu^{1}(t)\right) \geq \mathcal{E}\left(\mu^{2}(t)\right)+\int_{\mathcal{M} \times \mathcal{M}} g\left(\kappa^{2}(t, y), T(y, x)\right) d \gamma_{t}(x, y)+\frac{\lambda}{2} d_{W}^{2}\left(\mu^{1}(t), \mu^{2}(t)\right) .
$$

Adding together gives

$$
-\lambda d_{W}^{2}\left(\mu^{1}(t), \mu^{2}(t)\right) \geq \int_{\mathcal{M} \times \mathcal{M}}\left(g\left(\kappa^{1}(t, x), T(x, y)\right) d \gamma_{t}(x, y)+g\left(\kappa^{2}(t, y), T(y, x)\right)\right) d \gamma_{t}(x, y) .
$$

By Lemma 4.3.4 from [2] and Lemma 5.3 we have

$$
\begin{aligned}
\frac{d}{d t} d_{W}^{2}\left(\mu^{1}(t), \mu^{2}(t)\right) & \leq-2 \int_{\mathcal{M} \times \mathcal{M}}\left(g\left(v^{1}(t, x), T(x, y)\right)+g\left(v^{2}(t, y), T(y, x)\right)\right) d \gamma_{t}(x, y) \\
& =2 \int_{\mathcal{M} \times \mathcal{M}}\left(g\left(\kappa^{1}(t, x), T(x, y)\right)+g\left(\kappa^{2}(t, y), T(y, x)\right)\right) d \gamma_{t}(x, y) \\
& \leq-2 \lambda d_{W}^{2}\left(\mu^{1}(t), \mu^{2}(t)\right)
\end{aligned}
$$

We can use Gronwall's inequality to get,

$$
d_{W}\left(\mu^{1}(t), \mu^{2}(t)\right) \leq e^{-\lambda t} d_{W}\left(\mu_{0}^{1}, \mu_{0}^{2}\right) .
$$

1.19 is proved.

Now we turn to the relationship between gradient flow and system of evolution variational inequalities. If $\mu^{1}(t)$ is a gradient flow with respect to $\mathcal{E}$, then by Lemma 5.3

$$
\begin{aligned}
\frac{1}{2} \frac{d}{d t} d_{W}^{2}\left(\mu^{1}(t), \nu\right) & =-\int_{\mathcal{M} \times \mathcal{M}} g_{x}\left(v^{1}(t, x), T(x, y)\right) \\
& =\int_{\mathcal{M} \times \mathcal{M}} g_{x}\left(\kappa^{1}(t, x), T(x, y)\right) \\
& \leq \mathcal{E}(\nu)-\mathcal{E}(\mu(t))-\frac{\lambda}{2} d_{W}^{2}(\mu(t), \nu),
\end{aligned}
$$

for a.e. $t>0$, which implies the system of evolution variational inequalities. If $\mu^{1}(t)$ satisfies the system of evolution variational inequalities 1.20 , then

$$
\begin{aligned}
\frac{1}{2} \frac{d}{d t} d_{W}^{2}\left(\mu^{1}(t), \nu\right) & =-\int_{\mathcal{M} \times \mathcal{M}} g_{x}\left(v^{1}(t, x), T(x, y)\right) d \gamma_{t}(x, y) \\
& \leq \mathcal{E}(\nu)-\mathcal{E}\left(\mu^{1}(t)\right)-\frac{\lambda}{2} d_{W}^{2}\left(\mu^{1}(t), \nu\right) .
\end{aligned}
$$

By the definition of subdifferential of $\mathcal{E}$, we know that $v^{1}(t) \in-\partial \mathcal{E}\left(\mu^{1}(t)\right)$ for a.e. $t>0$, and thus $\mu^{1}(t)$ is a gradient flow with respect to $\mathcal{E}$.

Thus gradient flow is characterized by the system of evolution variational inequalities. 


\section{6. $\lambda$-GEODESIC CONVEXITY OF $\mathcal{E}$}

In this section, we present the details on obtaining conditions on $g, W, V$ to guarantee $\lambda$-geodesic convexity of $W, V$ and thus $\mathcal{E}$. We also give some examples of Riemannian manifold $(\mathcal{M}, g)$, on which we derive explicit conditions on $W, V$ for $\mathcal{E}$ to be $\lambda$-geodesically convex. In particular we consider examples which explore how far can the conditions for $\lambda$-convexity be extended. Let us also mention that the general conditions when only the external potential, $V$, is present follow from the work of Sturm [40, who studied them together with internal energy.

We start by deriving the general formula of $\frac{d^{2}}{d t^{2}} \mathcal{E}(\mu(t))$ for $\mu(t)$ geodesics in $\mathcal{P}_{2}(\mathcal{M})$. Notice that we only need the existence of optimal plans between $\mu, \nu \in \mathcal{P}_{2}(\mathcal{M})$ and then the interpolation of optimal plans is a geodesic. By [45], Corollary 7.22, we know that geodesics starting from $\mu$ in $\mathcal{P}_{2}(\mathcal{M})$ are of the form

$$
\mu(t)=\left(F_{t}\right)_{\sharp} \mu
$$

where $F_{t}(x)=\exp _{x}(t \nabla \phi)$ is the geodesic on $\mathcal{M}$. We write $x_{t}=F_{t}(x)$, for simplicity. By definition of push forward of measures,

$$
\mathcal{E}(\mu(t))=\mathcal{W}(\mu(t))+\mathcal{V}(\mu(t))=\frac{1}{2} \int_{\mathcal{M} \times \mathcal{M}} W\left(x_{t}, y_{t}\right) d \mu(x) d \mu(y)+\int_{\mathcal{M}} V\left(x_{t}\right) d \mu(x) .
$$

Since $x_{t}$ and $y_{t}$ are geodesics on $\mathcal{M},\left(x_{t}, y_{t}\right)$ is a geodesic on the product manifold $\mathcal{M} \times \mathcal{M}$. When $W, V$ are twice differentiable direct computation shows:

$$
\begin{aligned}
\frac{d^{2}}{d t^{2}} \mathcal{E}(\mu(t))= & \int_{\mathcal{M}} \operatorname{Hess}_{\mathcal{M}} V\left(x_{t}\right)\left(\dot{x}_{t}, \dot{x}_{t}\right) d \mu(x) \\
& +\frac{1}{2} \int_{\mathcal{M} \times \mathcal{M}} \operatorname{Hess}_{\mathcal{M} \times \mathcal{M}} W\left(x_{t}, y_{t}\right)\left(\dot{x}_{t}, \dot{y}_{t}\right)\left(\dot{x}_{t}, \dot{y}_{t}\right) d \mu(x) d \mu(y)
\end{aligned}
$$

where Hess $\mathcal{M}_{\mathcal{M}}, \operatorname{Hess}_{\mathcal{M} \times \mathcal{M}}$ are Hessian on $(\mathcal{M}, g)$ and $(\mathcal{M} \times \mathcal{M}, g \times g)$. So to verify convexity it suffices to show that there exists $\lambda \in \mathbb{R}$ such that for all vector fields $\dot{x}_{t}$ as above that

$$
\frac{d^{2}}{d t^{2}} \mathcal{E}(\mu(t)) \geq \lambda \int_{\mathcal{M}} g\left(\dot{x}_{t}, \dot{x}_{t}\right) d \mu(x) \text {. }
$$

So in general, $\lambda$-geodesic convexity of $V$ on $(\mathcal{M}, g)$ and $W$ on $(\mathcal{M} \times \mathcal{M}, g \times g)$ implies $\lambda$-geodesic convexity of $\mathcal{E}$. Actually, by [40], the potential energy $\mathcal{V}$ is $\lambda$-geodesic convex if and only if $\operatorname{Hess}_{\mathcal{M}} V \geq \lambda g$. Since $\mathcal{M}$ is a subset of $\mathbb{R}^{d}$ and $W(x, y)=W(x-y)$, we can expand $\frac{d^{2}}{d t^{2}} \mathcal{E}(\mu(t))$ in local coordinates,

$$
\begin{aligned}
\frac{d^{2}}{d t^{2}} \mathcal{E}(\mu(t))= & \frac{1}{2} \int_{\mathcal{M} \times \mathcal{M}}\left(\operatorname{Hess} W\left(x_{t}, y_{t}\right)\left(\dot{x}_{t}, \dot{y}_{t}\right)\left(\dot{x}_{t}, \dot{y}_{t}\right)\right. \\
& \left.+\sum_{k, i, j} \frac{\partial W}{\partial z_{k}}\left(x_{t}-y_{t}\right)\left(-\Gamma_{i j}^{k}\left(x_{t}\right)\left(\dot{x}_{t}\right)_{i}\left(\dot{x}_{t}\right)_{j}+\Gamma_{i j}^{k}\left(y_{t}\right)\left(\dot{y}_{t}\right)_{i}\left(\dot{y}_{t}\right)_{j}\right)\right) d \mu(x) d \mu(y) \\
& +\int_{\mathcal{M}}\left(\operatorname{Hess} V\left(x_{t}\right)\left(\dot{x}_{t}, \dot{y}_{t}\right)+\sum_{k, i, j} \frac{\partial V}{\partial z_{k}}\left(x_{t}\right)(-1) \Gamma_{i j}^{k}\left(x_{t}\right)\left(\dot{x}_{t}\right)_{i}\left(\dot{x}_{t}\right)_{j}\right) d \mu(x)
\end{aligned}
$$

where $\Gamma_{i j}^{k}$ are the Christoffel symbols on $(\mathcal{M}, g)$. This verifies the simple conditions we give in Section 1. Indeed, using that Hess $\mathcal{M} V_{i j}=\operatorname{Hess} V_{i j}-\frac{\partial V}{\partial z_{k}} \Gamma_{i j}^{k}$ and $\Gamma_{i j}^{k}=\frac{1}{2} A_{k m}\left(\frac{\partial G_{m i}}{\partial x_{j}}+\frac{\partial G_{m j}}{\partial x_{i}}-\frac{\partial G_{i j}}{\partial x_{m}}\right)$, the formula (6.3) allows us to conclude:

- If $(\mathcal{M}, g)$ is geodesically convex and compact with $G \in C^{1}(\mathcal{M})$, then any $V \in C^{2}(\mathcal{M})$ is $\lambda$-geodesically convex and $W \in C^{2}\left(\mathbb{R}^{d}\right)$ is $\lambda$-geodesically convex. Indeed, Hess $V_{i j}, \nabla V$ and $\Gamma_{i j}^{k}$ are bounded on $\mathcal{M}$, so $\operatorname{Hess}_{\mathcal{M}} V \geq C I_{d} \geq \tilde{C} G$ for all $x \in \mathcal{M}$. 
- If $g$ is $C^{1}$ bounded from below with bounded first derivative, and $V \in C^{2}(\mathcal{M})$ with bounded first and second derivative, then $V$ is $\lambda$-geodesically convex on $(\mathcal{M}, g)$.

- If $g$ is $C^{1}$ bounded from below and $V \in C^{2}(\mathcal{M})$ with Hess $V \geq c I_{d}$ such that $\Gamma_{i j}^{k} \frac{\partial V}{\partial z_{k}}$ is bounded from above on $\mathcal{M}$, then $V$ is $\lambda$-geodesically convex.

One obtains similar conditions on $W$ :

- If $g$ is $C^{1}$ bounded from below with bounded first derivative, and $W \in C^{2}(\mathcal{M})$ with bounded first and second derivative, then $W(x, y)=W(x-y)$ is $\lambda$-geodesically convex on $(\mathcal{M} \times \mathcal{M}, g \times$ $g)$.

- If $(\mathcal{M}, g)$ is geodesically convex and compact with $g \in C^{1}(\mathcal{M})$, then for any $W$ twice differentiable with Hess $W(y) \geq-c I_{d}$ for all $y \in \mathcal{M}-\mathcal{M}=\left\{x^{1}-x^{2}: x^{1} \in \mathcal{M}, x^{2} \in \mathcal{M}\right\}$ and some constant $c>0$. Note that since $\mathcal{M}$ is compact, $g \in C^{1}(\mathcal{M})$ and $W$ twice differentiable imply there exist constants $c_{1}>0, c_{2}>0$ such that $c_{1} I_{d} \leq G(x) \leq \frac{1}{c_{1}} I_{d}$, $\left|\frac{\partial}{\partial x_{k}} G_{i j}\right| \leq \frac{1}{c_{1}}$ and $|\nabla W(y)| \leq c_{2}$ for all $x \in \mathcal{M}$ and $y \in \mathcal{M}-\mathcal{M}, W$ is $\lambda$-geodesically convex on $(\mathcal{M} \times \mathcal{M}, g \times g)$. In particular, any $W \in C^{2}\left(\mathbb{R}^{d}\right)$ is $\lambda$-geodesically convex on $(\mathcal{M} \times \mathcal{M}, g \times g)$ for $(\mathcal{M}, g)$ geodesically convex and compact with $g \in C^{1}(\mathcal{M})$.

Note that the coupling between $\nabla W$ and $\Gamma_{i j}^{k}$ is of the form $\frac{\partial W}{\partial z_{k}}(x-y) \Gamma_{i j}^{k}(x)$, so we do not have the same conditions as the second item for the $\lambda$-geodesic convexity of $V$. This coupling prevents us from getting some simple conditions of $W, g$ to ensure $\lambda$-geodesic convexity of $W$, even in the 1-D case. It is more transparent in the 1-D examples of $W$, Example 6.3 .

We now investigate conditions on $V, W$. Let us first focus on potential $V$ :

Example 6.1. Consider $d=1$ and $(\mathcal{M}, g)=\left(\mathbb{R}_{+}^{1}, g(x)\right)$, then conditions for $\lambda$-geodesic convexity of $V$ is

$$
V^{\prime \prime}(x)-\frac{g^{\prime}(x)}{2 g(x)} V^{\prime}(x) \geq \lambda g(x) .
$$

- $g(x)=x^{p}$ for some $p<0$, then $V(x)=V_{0}+\int_{1}^{x} y^{\frac{p}{2}} U(y) d y$ is $\lambda$-geodesically convex if $U \in C^{1}\left(\mathbb{R}_{+}^{1}\right)$ with $x^{-\frac{p}{2}} U^{\prime}(x) \geq C$ for all $x>0$ and some constant $C$. Moreover, $V$ is geodesically convex if $U^{\prime}(x) \geq 0$ for all $x>0$. In particular, it is straightforward to check $V(x)=x^{q}$ for $q \geq \max \left\{0, \frac{p}{2}+1\right\}$ or $q \leq \min \left\{0, \frac{p}{2}+1\right\}$ is geodesically convex. Indeed, 6.4 becomes

$$
V^{\prime \prime}(x)-\frac{p}{2 x} V^{\prime}(x) \geq \lambda x^{p}
$$

which is

$$
\left(x^{-\frac{p}{2}} V^{\prime}(x)\right)^{\prime} \geq \lambda x^{\frac{p}{2}}
$$

for $x>0$. Since $U(x)=x^{-\frac{p}{2}} V^{\prime}(x)$, the last condition becomes $U^{\prime}(x) \geq \lambda x^{\frac{p}{2}}$. So for any $U \in C^{1}\left(\mathbb{R}_{+}^{1}\right)$ with $x^{-\frac{p}{2}} U^{\prime}(x) \geq C$ for some constant $C, V(x)=V_{0}+\int_{1}^{x} y^{\frac{p}{2}} U(y) d y$ is $\lambda$-geodesically convex on $(\mathcal{M}, g)$. If $U^{\prime}(x) \geq 0$, then $V$ is geodesically convex on $(\mathcal{M}, g)$.

- $g(x)=e^{\frac{p}{x}}$ for some $p>0$, then $V=V_{0}+\int_{1}^{x} e^{\frac{p}{2 y}} U(y) d y$ is $\lambda$-geodesically convex on $(\mathcal{M}, g)$, if $U \in C^{1}\left(\mathbb{R}_{+}^{1}\right)$ with $e^{-\frac{p}{2 x}} U^{\prime}(x) \geq C$ for all $x>0$ and some constant $C$. If $U^{\prime}(x) \geq 0$ for all $x>0$, then $V$ is geodesically convex on $(\mathcal{M}, g)$. In particular, $V(x)=x^{q}$ is geodesically convex for $q \geq 1$ and $\lambda$-geodesically convex for $q<1$. Similarly to the above case, the differential inequality (6.4) becomes

$$
V^{\prime \prime}(x)+\frac{p}{2 x^{2}} V^{\prime}(x) \geq \lambda e^{\frac{p}{x}},
$$

which implies

$$
\left(e^{-\frac{p}{2 x}} V^{\prime}(x)\right)^{\prime} \geq \lambda e^{\frac{p}{2 x}}
$$


for all $x>0$. Take $U(x)=e^{-\frac{p}{2 x}} V^{\prime}(x)$, we have $U^{\prime}(x) \geq \lambda e^{\frac{p}{2 x}}$ and $V(x)=V_{0}+\int_{1}^{x} e^{\frac{p}{2 x}} U(y) d y$. Notice that for any $U \in C^{1}\left(\mathbb{R}_{+}\right)$with $U^{\prime}(x) \geq C$ for some constant $C$, we have there exists $\lambda \in \mathbb{R}$ such that $U^{\prime}(x) \geq \lambda e^{\frac{p}{2 x}}$ since $e^{\frac{p}{2 x}}$ is bounded from below. And if $U^{\prime}(x) \geq 0$ we can take $\lambda=0$. So for any $U \in C^{1}\left(\overline{\mathbb{R}_{+}}\right)$, such that $U^{\prime}$ is bounded from below, then $V(x)=V(0)+\int_{0}^{x} e^{\frac{p}{2 y}} U(y) d y$ is $\lambda$-geodesically convex on $(\mathcal{M}, g)$.

Example 6.2. Consider the upper half space, $\mathbb{R}^{d-1} \times[0, \infty)$ endowed with a Riemannian metric given by

$$
G(x)=\left[\begin{array}{cc}
g\left(x_{d}\right) I_{d-1} & 0 \\
0 & 1
\end{array}\right]
$$

Then

$$
\Gamma_{i j}^{k}= \begin{cases}\frac{1}{2} g^{-1}\left(x_{d}\right) g^{\prime}\left(x_{d}\right) & \text { if }\{i, j\}=\{k, d\}, k<d, \\ -\frac{1}{2} g^{\prime}\left(x_{d}\right) & \text { if } i=j<d, k=d, \\ 0 & \text { otherwise. }\end{cases}
$$

Let $\mathcal{M}$ be a compact, geodesically convex subset of $\mathbb{R}_{+}^{d}$ with $C^{1}$ boundary. For any $V \in C^{2}\left(\mathbb{R}_{+}^{d}\right), W \in$ $C^{2}\left(\mathbb{R}^{d}\right), V, W$ are $\lambda$-geodesically convex on $(\mathcal{M}, g)$ and $(\mathcal{M} \times \mathcal{M}, g \times g)$.

Consider now $d=2$ and $g\left(x_{2}\right)=x_{2}^{p}$ with $p<0$. For simplicity, we assume that $\mathcal{M}$ contains portion of $x_{2}=0$. We note that the metric is degenerate. Nevertheless investigate if $V(x)=|x|^{2}$ should be $\lambda$-convex in some generalized sense. Direct computation shows

$$
\operatorname{Hess}_{\mathcal{M}} V(x)=\left[\begin{array}{cc}
2+p x_{2}^{p} & -p x_{1} x_{2}^{-1} \\
-p x_{1} x_{2}^{-1} & 2
\end{array}\right]
$$

For $V$ to be $\lambda$-convex it is necessary that

$$
2 \geq \lambda
$$

and

$$
(2-\lambda)\left(2+(p-\lambda) x_{2}^{p}\right)-p^{2} x_{1}^{2} x_{2}^{-2} \geq 0 .
$$

By taking $x_{2} \rightarrow 0^{+}$shows that no $\lambda \in \mathbb{R}$ can satisfy these conditions.

In general the conditions for the $\lambda$-geodesic convexity of $V$ and $W$ are rather restrictive, as claimed in Remark 1.1. The next example illustrates why.

Example 6.3. Take $(\mathcal{M}, g)$ to be $(\mathbb{R}, g)$. Then the $\lambda$-geodesic convexity condition for $W$ is

$$
\left[\begin{array}{cc}
W^{\prime \prime}(x-y)-\frac{1}{2} W^{\prime}(x-y) g^{-1}(x) g^{\prime}(x) & -W^{\prime \prime}(x-y) \\
-W^{\prime \prime}(x-y) & W^{\prime \prime}(x-y)+\frac{1}{2} W^{\prime}(x-y) g^{-1}(y) g^{\prime}(y)
\end{array}\right] \geq \lambda\left[\begin{array}{cc}
g(x) & 0 \\
0 & g(y)
\end{array}\right] .
$$

In particular it is necessary that for all $x, y \in \mathbb{R}$

$$
W^{\prime \prime}(x-y)-\frac{1}{2} W^{\prime}(x-y) g^{-1}(x) g^{\prime}(x) \geq \lambda g(x) .
$$

One should contrast this condition with condition 6.4 for potential $V$. In particular the condition above shows the presence of long-range effects which make it hard the condition to be satisfied. For example, if $W(z)=z^{2}$, and $g(z)=2+\frac{\sin (z)}{1+z^{2}}$ then the condition above becomes

$$
2-(x-y) \frac{\left(1+x^{2}\right) \cos (x)-2 x \sin (x)}{2\left(1+x^{2}\right)+\sin x} \geq \lambda g(x)
$$

taking $x$ such that the term next to $(x-y)$ is negative and then taking $y \rightarrow \infty$ shows that there is no $\lambda$ for which the condition is satisfied.

Nevertheless a usable sufficient condition for $\lambda$-convexity can be found. For example $W \in C^{2}(\mathbb{R})$, with $w$ even, $w^{\prime}, w^{\prime \prime}$ bounded, $g \in C^{1}(\overline{\mathbb{R}})$ with $g \geq C>0$ and $g^{\prime}$ bounded suffices. 
Acknowledgement. The authors are grateful to José Antonio Carrillo, Alessio Figalli for stimulating discussions and David Kinderlehrer for valuable suggestions and constant support. LW acknowledges the support from NSF DMS 0806703. DS is also grateful to NSF (grant DMS-0908415). The research was also supported by NSF PIRE grant OISE-0967140. Authors are thankful to the Center for Nonlinear Analysis (NSF grant DMS-0635983) for its support.

\section{REFERENCES}

[1] L. Ambrosio and N. Gigli, A user's guide to optimal transport, in Modelling and Optimisation of Flows on Networks, B. Piccoli and M. Rascle, eds., Lecture Notes in Mathematics, Springer Berlin Heidelberg, Berlin Heidelberg, 2013.

[2] L. Ambrosio, N. Gigli, And G. Savaré, Gradient flows in metric spaces and in the space of probability measures, Lectures in Mathematics ETH Zürich, Birkhäuser Verlag, Basel, second ed., 2008.

[3] - Heat flow and calculus on metric measure spaces with Ricci curvature bounded below-the compact case, Boll. Unione Mat. Ital. (9), 5 (2012), pp. 575-629.

[4] D. Balagué, J. A. Carrillo, T. Laurent, and G. Raoul, Dimensionality of local minimizers of the interaction energy, Arch. Ration. Mech. Anal., 209 (2013), pp. 1055-1088.

[5] D. Balagué, J. A. Carrillo, and Y. Yao, Confinement for repulsive-attractive kernels. Preprint, 2012.

[6] A. J. Bernoff and C. M. Topaz, A primer of swarm equilibria, SIAM J. Appl. Dyn. Syst., 10 (2011), pp. 212250.

[7] A. L. Bertozzi and J. Brandman, Finite-time blow-up of $L^{\infty}$-weak solutions of an aggregation equation, Commun. Math. Sci., 8 (2010), pp. 45-65.

[8] A. L. Bertozzi, J. A. Carrillo, and T. Laurent, Blow-up in multidimensional aggregation equations with mildly singular interaction kernels, Nonlinearity, 22 (2009), pp. 683-710.

[9] A. L. Bertozzi, J. B. Garnett, and T. Laurent, Characterization of radially symmetric finite time blowup in multidimensional aggregation equations, SIAM J. Math. Anal., 44 (2012), pp. 651-681.

[10] A. L. Bertozzi and T. Laurent, Finite-time blow-up of solutions of an aggregation equation in $\mathbf{R}^{n}$, Comm. Math. Phys., 274 (2007), pp. 717-735.

[11] A. L. Bertozzi, T. Laurent, and J. Rosado, $L^{p}$ theory for the multidimensional aggregation equation, Comm. Pure Appl. Math., 64 (2011), pp. 45-83.

[12] M. Burger and M. Di Francesco, Large time behavior of nonlocal aggregation models with nonlinear diffusion, Netw. Heterog. Media, 3 (2008), pp. 749-785.

[13] J. A. Carrillo, M. Di Francesco, A. Figalli, T. Laurent, and D. SlepČev, Global-in-time weak measure solutions and finite-time aggregation for nonlocal interaction equations, Duke Math. J., 156 (2011), pp. 229-271.

[14] _ Confinement in nonlocal interaction equations, Nonlinear Anal., 75 (2012), pp. 550-558.

[15] J. A. CARrillo ANd J. Rosado, Uniqueness of bounded solutions to aggregation equations by optimal transport methods, in European Congress of Mathematics, Eur. Math. Soc., Zürich, 2010, pp. 3-16.

[16] D. Cordero-Erausquin, R. J. McCann, and M. SchmuckenschläGer, A Riemannian interpolation inequality à la Borell, Brascamp and Lieb, Invent. Math., 146 (2001), pp. 219-257.

[17] S. Daneri and G. Savaré, Eulerian calculus for the displacement convexity in the Wasserstein distance, SIAM J. Math. Anal., 40 (2008), pp. 1104-1122.

[18] M. R. D’Orsogna, Y. L. Chuang, A. L. Bertozzi, and L. S. Chayes, Self-propelled particles with soft-core interactions: Patterns, stability, and collapse, Phys. Rev. Lett., 96 (2006), p. 104302.

[19] M. ERBAR, The heat equation on manifolds as a gradient flow in the Wasserstein space, Ann. Inst. Henri Poincaré Probab. Stat., 46 (2010), pp. 1-23.

[20] A. Fathi And A. Figalli, Optimal transportation on non-compact manifolds, Israel J. Math., 175 (2010), pp. 159.

[21] K. Fellner and G. Raoul, Stable stationary states of non-local interaction equations, Math. Models Methods Appl. Sci., 20 (2010), pp. 2267-2291.

$[22] \_$, Stability of stationary states of non-local equations with singular interaction potentials, Math. Comput. Modelling, 53 (2011), pp. 1436-1450.

[23] R. C. Fetecau, Y. Huang, and T. Kolokolnikov, Swarm dynamics and equilibria for a nonlocal aggregation model, Nonlinearity, 24 (2011), pp. 2681-2716.

[24] A. Figalli, Y.-H. Kim, and R. MCCAnn, Regularity of optimal transport maps on multiple products of spheres, J. Eur. Math. Soc. (JEMS), 15 (2013), pp. 1131-1166.

[25] I. Fonseca And G. Leoni, Modern methods in the calculus of variations: $L^{p}$ spaces, Springer Monographs in Mathematics, Springer, New York, 2007. 
[26] N. Gigli, On the heat flow on metric measure spaces: existence, uniqueness and stability, Calc. Var. Partial Differential Equations, 39 (2010), pp. 101-120.

[27] N. Gigli, K. Kuwada, And S.-I. Ohta, Heat flow on Alexandrov spaces, Comm. Pure Appl. Math., 66 (2013), pp. 307-331.

[28] Y. Huang And A. L. Bertozzi, Self-similar blowup solutions to an aggregation equation in $\mathbf{R}^{n}$, SIAM J. Appl. Math., 70 (2010), pp. 2582-2603.

[29] - Asymptotics of blowup solutions for the aggregation equation, Discrete Contin. Dyn. Syst. Ser. B, 17 (2012), pp. 1309-1331.

[30] Y. Huang, T. P. Witelski, And A. L. Bertozzi, Anomalous exponents of self-similar blow-up solutions to an aggregation equation in odd dimensions, Appl. Math. Lett., 25 (2012), pp. 2317-2321.

[31] T. Kolokolnikov, H. Sun, D. Uminsky, and A. L. Bertozzi, Stability of ring patterns arising from twodimensional particle interactions, Phys. Rev. E, 84 (2011), p. 015203.

[32] T. Laurent, Local and global existence for an aggregation equation, Comm. Partial Differential Equations, 32 (2007), pp. 1941-1964.

[33] A. J. Leverentz, C. M. Topaz, and A. J. Bernoff, Asymptotic dynamics of attractive-repulsive swarms, SIAM J. Appl. Dyn. Syst., 8 (2009), pp. 880-908.

[34] S. LisINI, Nonlinear diffusion equations with variable coefficients as gradient flows in Wasserstein spaces, ESAIM Control Optim. Calc. Var., 15 (2009), pp. 712-740.

[35] R. J. McCann, Polar factorization of maps on Riemannian manifolds, Geom. Funct. Anal., 11 (2001), pp. 589608.

[36] S.-I. Оhта And K.-T. Sturm, Non-contraction of heat flow on Minkowski spaces, Arch. Ration. Mech. Anal., 204 (2012), pp. 917-944.

[37] F. Отto And M. Westdickenberg, Eulerian calculus for the contraction in the Wasserstein distance, SIAM J. Math. Anal., 37 (2005), pp. 1227-1255 (electronic).

[38] P. Petersen, Riemannian geometry, vol. 171 of Graduate Texts in Mathematics, Springer, New York, second ed., 2006.

[39] G. RAOul, Non-local interaction equations: Stationary states and stability analysis. Preprint, 2011.

[40] K.-T. Sturm, Convex functionals of probability measures and nonlinear diffusions on manifolds, J. Math. Pures Appl. (9), 84 (2005), pp. 149-168.

[41] C. M. Topaz, A. J. Bernoff, S. Logan, And W. Toolson, A model for rolling swarms of locusts, Eur. Phys. J. Special Topics, 157 (2008), pp. 93-109.

[42] C. M. Topaz AND A. L. BerTOzzI, Swarming patterns in a two-dimensional kinematic model for biological groups, SIAM J. Appl. Math., 65 (2004), pp. 152-174.

[43] C. M. Topaz, A. L. Bertozzi, and M. A. Lewis, A nonlocal continuum model for biological aggregation, Bull. Math. Biol., 68 (2006), pp. 1601-1623.

[44] C. M. Topaz, M. R. D'Orsogna, L. Edelstein-Keshet, and A. J. Bernoff, Locust dynamics: behavioral phase change and swarming, PLoS Comput. Biol., 8 (2012), pp. e1002642, 11.

[45] C. Villani, Optimal transport, vol. 338 of Grundlehren der Mathematischen Wissenschaften [Fundamental Principles of Mathematical Sciences], Springer-Verlag, Berlin, 2009. Old and new.

Department of Mathematical Sciences, Carnegie Mellon University, Pittsburgh, PA 15213 USA

E-mail address: lijiangw@andrew.cmu.edu

Department of Mathematical Sciences, Carnegie Mellon University, Pittsburgh, PA 15213 USA

E-mail address: slepcev@math.cmu.edu 